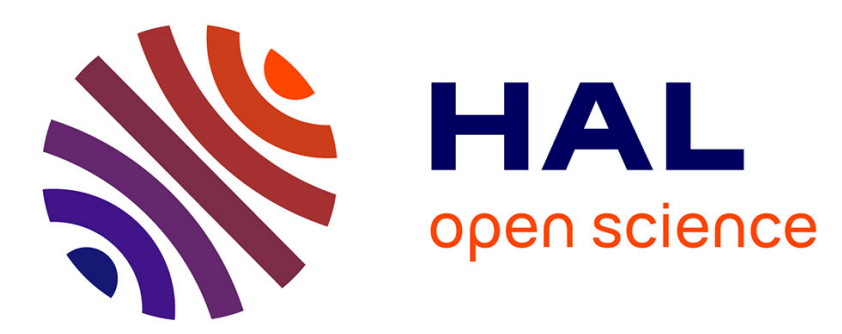

\title{
Improved modeling of the solid-to-plasma transition of polystyrene ablator for laser direct-drive inertial confinement fusion hydrocodes
}

\author{
A. Pineau, B. Chimier, S. Hu, G. Duchateau
}

\section{- To cite this version:}

A. Pineau, B. Chimier, S. Hu, G. Duchateau. Improved modeling of the solid-to-plasma transition of polystyrene ablator for laser direct-drive inertial confinement fusion hydrocodes. Physical Review E , 2021, 104 (1), 10.1103/PhysRevE.104.015210 . hal-03394721

HAL Id: hal-03394721

https://hal.science/hal-03394721

Submitted on 22 Oct 2021

HAL is a multi-disciplinary open access archive for the deposit and dissemination of scientific research documents, whether they are published or not. The documents may come from teaching and research institutions in France or abroad, or from public or private research centers.
L'archive ouverte pluridisciplinaire HAL, est destinée au dépôt et à la diffusion de documents scientifiques de niveau recherche, publiés ou non, émanant des établissements d'enseignement et de recherche français ou étrangers, des laboratoires publics ou privés. 


\title{
Improved modeling of the solid-to-plasma transition of polystyrene ablator for laser direct-drive inertial confinement fusion hydrocodes
}

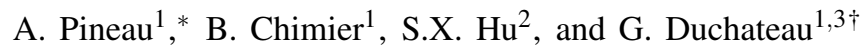 \\ ${ }^{1}$ Université de Bordeaux-CNRS-CEA, Centre Lasers Intenses et Applications, \\ UMR 5107, 351 Cours de la Libération, 33405 Talence Cedex, France \\ 2 Laboratory for Laser Energetics, University of Rochester, \\ 250 East River Road, Rochester, New York 14623, USA and \\ 3 CEA CESTA, 15 Avenue des Sablières, CS60001, 33116 Le Barp Cedex, France
}

(Dated: June 24, 2021)

\begin{abstract}
The target performance of laser direct-drive inertial confinement fusion (ICF) can be limited by the development of hydrodynamic instabilities resulting from the non-homegeneous laser absorption at the target surface, i.e. the laser imprint on the ablator. To understand and describe the formation of these instabilities, the early ablator evolution during the laser irradiation should be considered. In this work, an improved modeling of the solid-to-plasma transition of a polystyrene ablator for laser direct-drive ICF is proposed. This model is devoted to be implemented in hydrocodes dedicated to ICF which generally assume an initial plasma state. The present approach consists of the two-temperature model coupled to the electron, ion and neutral dynamics including the chemical fragmentation of polystyrene. The solid-to-plasma transition is shown to significantly influence the temporal evolution of both free electron density and temperatures, which can lead to different shock formation and propagation compared with an initial plasma state. The influence of the solid-to-plasma transition on the shock dynamics is evidenced by considering the scaling law of the pressure with respect to the laser intensity. The ablator transition is shown to modify the scaling law exponent compared with an initial plasma state.
\end{abstract}

\section{INTRODUCTION}

Laser direct-drive inertial confinement fusion consists in focusing high-energetic lasers onto a target in order to compress it and create nuclear fusion reactions [1-4]. Typical targets are usually made of a cryogenic deuterium-tritium (DT) shell filled with a gaseous DT mixture. The cryogenic DT shell is covered with a plastic layer, namely the ablator, which is often polystyrene [5]. By absorbing the laser energy, the ablator initially in a solid state is converted into a plasma and expands leading to the compression of the target through the so-called rocket effect. Intensity modulations coming from laser speckles or beam-to-beam interactions can lead to a non-homogeneous laser absorption and density modulations at the target surface, which can be seeds of Rayleigh-Taylor and Richtmyer-Meshkov instabilities, i.e. the laser-imprinting process [6]. The latter reduces drastically ICF target performances [7-9]. To understand and control the formation of such instabilities, the ablator evolution during the laser irradiation should be taken into account, especially the solid-toplasma transition.

The solid-to-plasma transition is generally neglected in ICF hydrocodes by assuming a negligible transition time compared with the total laser pulse duration. Simulations start with an ablator in the plasma state with an electron density higher than the critical density of the corresponding laser. However, a recent study has shown that the timescale of this transition is of the order of $100 \mathrm{ps}$ for a conventional laser pulse irradiation used in ICF [5], i.e. of the order of magnitude of the picket duration [10]. Moreover, the influence

* adrien.pineau@u-bordeaux.fr $\dagger$ guillaume.duchateau@cea.fr of the solid-to-plasma transition on the hydrodynamic procceses, resulting from the laser absorption, has been highlighted by measuring the laser induced shock velocities in polystyrene [11]. Significant differences have been observed between experimental results and hydrodynamic simulations where the solid-to-plasma transition is not considered. Indeed, in the plasma state, the relative variations of the pressure scales linearly with the laser intensity following a linear absorption process, whereas a non linear behavior has been shown to be necessary to account for experimental observations [11]. This non linear behavior has been attributed to the primary step of plasma formation through two-photon absorption of polystyrene.

A first microscopic modeling of the solid-to-plasma transition has been proposed for a polystyrene ablator [5]. The ablator heating eventually leading to phase transition and plasma formation is evaluated owing to a two-temperature model describing the coupled evolution of both electron and lattice temperatures, and including the free electron density evolution induced by the laser irradiation. The free electron density is estimated by describing processes of photoionization, electron impact ionization, and electron recombination. Although this first microscopic modeling laid the foundations of the description of the solid-to-plasma transition, it must be improved for its introduction in a hydrocode due to several reasons. (i) The densities of carbon and hydrogen ions are not predicted whereas they are required to describe properly the plasma formation. The chemical fragmentation of initial solid polystyrene leading to atomic species has thus to be described (ii) The description of electron collisions is rather crude despite it provides correct orders of magnitude. To predict accurate electron and lattice heating (leading to phase transitions), a more accurate description of collisional processes is required. (iii) In the perspective of a model properly describing the material decomposition into a plasma where various 
transient chemical species are involved (electrons, polymer, molecules, atoms), adapted equations of state (EOS) have to be implemented.

The first goal of the present work is to introduce an improved microscopic modeling of the solid-to-plasma transition where above mentioned three assumptions of the first modeling attempt are fixed. The chemical fragmentation and improved collision frequencies are introduced following developments proposed in [12]. Both the energy transfer and the laser induced electron dynamics including photoionization, electron impact ionization, and electron recombination, are adapted to the state of matter. These theoretical developments are presented in Section III. Section III] presents numerical simulations performed by using laser intensity profiles pertaining to ICF conditions. The solid-to-plasma transition is investigated where in particular the evolution of the chemical composition of the ablator is highlighted. Comparisons with typical initial conditions of ICF hydrocodes are also performed. The second goal of the present work is to address the evolution of the pressure as a function of the laser intensity following observations in [11]. A non linear behavior is evidenced in section $\mathrm{V}$ confirming the role of the solid-to-plasma transition on the subsequent plasma hydrodynamics. Finally, conclusions and outlooks are drawn in Sec. VI Owing to the fact it includes all main physical processes driving the solidto-plasma transition, and provides densities and temperatures of formed plasma, the present model is devoted to be introduced in current hydrocode to improve their accuracy and correctly describe the onset of hydrodynamics which is expected to influence the subsequent implosion of ICF targets.

\section{MODELING OF THE SOLID-TO-PLASMA TRANSITION}

The phenomenology of the laser induced phase transition of a polystyrene ablator, i.e. chemical fragmentation, and its laser interaction are first summarized following a previous work [12] in order to introduce the forthcoming theoretical developments. Let define $T_{i l}$ the temperature of the so called ion-lattice system [5] which includes various chemical species as polymer, molecules, or atoms with different ionization degree [12]. If $T_{i l}<58 \mathrm{meV}$, the ablator is constituted of solid polystyrene which is a polymer. In that case, there is a band structure constraining the electron dynamics, and the electrons undergo collisions with phonons. If $T_{i l}>58 \mathrm{meV}$, the ablator consists of several species, namely styrene, benzene, acetylene molecules, or carbon and hydrogen atoms, with various ionization degrees, depending on temperatures. In that case, the electron dynamics consists of successive ionizations of these molecules and atoms. Within this phase, electrons undergo collisions with ions and neutral species. As a final product of the laser induced and thermal molecule dissociations, the ablator consists of a mixture of ionized carbon and hydrogen atomic species (i.e. at the end of the solid-to-plasma transition). Such a scenario for the chemical fragmentation of polystyrene implies that laser induced electron dynamics, electron collision frequencies, and energy transfer between various species populations, have to be adapted to a given chemical composition which depends on ion-lattice temperature.

For typical laser pickets used in ICF experiments, i.e. Gaussian profile with a maximum laser intensity about $10^{14}$ $\mathrm{W} . \mathrm{cm}^{-2}$ and a duration of $100 \mathrm{ps}$, the solid-to-plasma takes place over a timescale of about $100 \mathrm{ps}$ [5] which is smaller than the hydrodynamic timescale. Hydrodynamic processes can thus be neglected a priori (based on the forthcoming results, an estimation of the hydrodynamic expansion will confirm this assumption a posteriori). In addition, thermal diffusion is also neglected. The smallest spatial scale where absorption is not uniform corresponds to the intensity profile of one laser speckle which characteristic size is of the order of $1 \mu \mathrm{m}$. Due to the variations of absorption and energy deposition on this spatial scale, the resulting temperature gradient may lead to thermal diffusion in general. By using the expression of thermal conductivity provided in [12], calculations show that the characteristic diffusion time becomes short enough ( $\sim 1 \mathrm{ps})$ to induce heat diffusion only when the solidto-plasma transition is finished, i.e. when a fully plasma state is formed. Heat diffusion thus can be neglected during the solid-to-plasma transition.

The ablator is a system out of equilibrium (temperatures of electrons and ion-lattice are different in general) during the solid-to-plasma transition since electrons are first excited by the laser, and the ion-lattice system is then heated subsequenly through electron collisions which transfer their energy. The evolution of the electron and ion-lattice temperatures $\left(T_{e}\right.$ and $T_{i l}$, respectively) is modeled with the two-temperature model which reads:

$$
\begin{aligned}
C_{e} \frac{\partial T_{e}}{\partial t} & =-\tilde{\mu}_{e} \frac{\partial n_{e}}{\partial t}-\left(\frac{\partial U_{e}}{\partial t}\right)_{\text {collisions }}+\left(\frac{\partial U_{e}}{\partial t}\right)_{\text {laser }} \\
C_{i l} \frac{\partial T_{i l}}{\partial t} & =-\sum_{s} \tilde{\mu}_{s} \frac{\partial n_{s}}{\partial t}+\left(\frac{\partial U_{e}}{\partial t}\right)_{\text {collisions }}
\end{aligned}
$$

where ions and neutral species are assumed to have the same temperature. $C_{e}$ and $C_{i l}$ correspond to the electron and ionlattice heat capacities, respectively. The first term in the right hand side of both equations correspond to the variation of energy due to the evolution of the number of particules $\left(\tilde{\mu}_{e}\right.$ and $\tilde{\mu}_{s}$ are effective chemical potentials and the sum is over the different chemical species constituting the ion-lattice system, see Appendix A). The second terms account for the energy transfer from the electrons to the ion-lattice system due to electron collisions. The last term in Eq. 1 corresponds to the laser heating. Except $C_{e}$ and $\left(\partial_{t} U_{e}\right)_{\text {laser }}$ which exhibit generic expression, all the quantities appearing in Eqs. (1) and (2) depend on the chemical composition of the ablator and have to be defined according to the ion-lattice temperature. 


\section{A. Laser heating}

The laser energy absorbed by electrons $\left(\partial_{t} U_{e}\right)_{\text {laser }}$ is given by the Drude model [5]:

$$
\left(\frac{\partial U_{e}}{\partial t}\right)_{\text {laser }}=\frac{e^{2} n_{e} v_{c}}{m_{e}\left(\omega_{L}^{2}+v_{c}^{2}\right)} E_{L}^{2}
$$

where $\omega_{L}$ and $E_{L}$ are the laser frequency and laser electric field, respectively. The electron collision frequency $v_{c}$ is given by a model dedicated to the solid-to-plasma transition of the polystyrene ablator [12]. If the lattice-ion temperature is smaller than $58 \mathrm{meV}, v_{c}$ is evaluated from collisions between electrons and both acoustic and optical phonons of solid polystyrene. This collision frequency is evaluated with the Fermi golden rule. If the lattice-ion temperature is higher than $58 \mathrm{meV}$, electron-ion collisions take place, which are described by the Lee \& More model [13] using the average collision time. A Coulomb logarithm different from the Lee \& More model is used [14] since collisions may take place in a strongly coupled regime for electron temperatures of a few $\mathrm{eV}$. We have checked by evaluating the electron and ion coupling parameter that such an estimation for the Coulomb logarithm is suitable for the solid-to-plasma transition. Electron-neutral collisions are described by using classical cross sections.

\section{B. Equations of state}

The electron heat capacity $C_{e}$ and the effective chemical potential $\tilde{\mu}_{e}$ are evaluated for both $T_{i l}<58 \mathrm{meV}$ and $T_{i l}>58$ $\mathrm{meV}$ from the general free electron gas equation of state which includes both degenerated limit and ideal gas behavior for temperatures larger than the Fermi temperature (see Appendix B). The ion-lattice heat capacity $C_{i l}$ and the ionlattice effective chemical potential $\tilde{\mu}_{s}$ depend on the considered species and thus depend on the ion-lattice temperature.

For $T_{i l}<58 \mathrm{meV}, C_{i l}$ is the heat capacity of solid polystyrene. In that case, $C_{i l}=C_{m} \rho / M$ with $M=104$ g.mol ${ }^{-1}$ and $\rho=1.1 \mathrm{~g} . \mathrm{cm}^{-3}$ the styrene molecular mass and polystyrene density, respectively. The molar heat capactiy $C_{m}$ of polystyrene, which depends on the lattice temperature, is given by a fit of experimental data [15]. $\tilde{\mu}_{s}$ is associated to the variation of the polystyrene energy due to the variation of the phonon density. Because the internal energy of polystyrene does not depend on the phonon density [16], it comes directly $\tilde{\mu}_{s}=0$.

For $T_{i l}>58 \mathrm{meV}, C_{i l}$ corresponds to the heat capacity of both ions and neutrals (molecular and atomic species). Because a same temperature is assumed, $C_{i l}=C_{i}+C_{n}$ with $C_{i}$ and $C_{n}$ the heat capacity of ions and neutrals, respectively. $C_{i}$ is deduced from the Cowan model [17] which is a standard equation of state used in ICF. For neutrals, an ideal gas is assumed, i.e. $C_{n}=3 k_{B} n_{n} / 2$ with $n_{n}$ the total density of neutrals.

The effective chemical potential of ions and neutrals, $\tilde{\mu}_{s}$, is given from the same EOS as the one used for the heat capacities, see Appendix A.

Note that relatively simple EOS are used in this model, in particular for neutrals. Forthcoming results of Sec. III shows that neutrals exist only during a short period of time. Thus, a more suitable EOS for neutrals is not expected to change the results and conclusions of this work. For electrons and ions, the thermal energy was compared to the Fermi energy and the interaction potential. It turned out that screening and quantum effects can be neglected during the solid-to-plasma transition. In addition, the presently used EOS have been compared (results not shown) with $a b$ initio calculations [18] providing the evolution of the pressure with respect to the temperature at equilibrium $\left(T_{e}=T_{i}\right)$. A good agreement is obtained, definitively confirming the EOS used in this model is suitable for the description of the solid-to-plasma transition.

\section{Electron dynamics}

Photoionization, electron impact ionization, and electron recombination are general processes taking place for all matter composition: polymer, molecules, or atoms. However the formalism used to describe these processes depends on the transient chemical composition, i.e. on the ion-lattice temperature $T_{i l}$. When the ablator species are molecules and atoms ionized or not, the dissociation of molecules has further to be considered.

\section{Conditions where $T_{i l}<58 \mathrm{meV}$}

The ablator is solid polystyrene which is a polymer, i.e. a dielectric material. The band structure consists of two bands, the valence band (VB) and the conduction band (CB), separated by a bandgap of $4.05 \mathrm{eV}$ [19]. Before any interaction, most of the electrons are in the VB while a small number is in the $\mathrm{CB}$. Because a polystyrene target with a temperature different from zero is considered, the CB is initially populated with an electron density of the order of $10^{10} \mathrm{~cm}^{-3}$ [20]. Due to photoionization, electrons in the $\mathrm{VB}$ are promoted into the $\mathrm{CB}$. Promoted electrons in the CB may reach higher energy levels by successive one-photon absorption. When the energy level becomes higher than the bandgap energy, those electrons may collide valence electrons resulting in two electrons in the bottom of the CB. The latter process is the so called electron impact ionization in dielectric materials [21]. In solid polystyrene, three levels of the CB have thus to be considered [5]. Accounting for these two ionization processes and electron recombination, the laser induced electron dynamics can be described as follows [5]:

$$
\begin{aligned}
& \frac{\partial n_{0}}{\partial t}=\alpha W_{P I}+2 \alpha v_{I I} n_{2}-W_{1} n_{0}-v_{r}\left(n_{0}-n_{c b 0}\right) \\
& \frac{\partial n_{1}}{\partial t}=W_{1} n_{0}-W_{1} n_{1}-v_{r} n_{1} \\
& \frac{\partial n_{2}}{\partial t}=W_{1} n_{1}-\alpha v_{I I} n_{2}-v_{r} n_{2}
\end{aligned}
$$

where $n_{0}, n_{1}$ and $n_{2}$ correspond to the electron densities in the three levels considered in the $\mathrm{CB}$ and $n_{c b 0}$ the initial electron density of the CB. $v_{I I}=10^{14} \mathrm{~s}^{-1}$ and $v_{r}=10^{12} \mathrm{~s}^{-1}$ 
correspond to the electron impact ionization and recombination frequencies, respectively. The photoionization rate $W_{P I}$ is evaluated with the Keldysh theory accounting for both tunnel ionization and multiphoton absorption [22]. The onephoton absorption rate is set to $W_{1}=10^{-7} E_{L}^{2}$, in units of $s^{-1}$, where $E_{L}$ is the laser electric field [5]. Finally, $\alpha=$ $1-\left(n_{0}+n_{1}+n_{2}\right) / n_{v b 0}$, with $n_{v b 0}$ the initial electron density in the VB, accounts for the depletion of the VB.

\section{Conditions where $T_{i l}>58 \mathrm{meV}$}

For this range of temperatures, the electron dynamics corresponds to the successive ionizations and dissociations of the molecular and atomic species. Within the ICF framework, the chemical species involved in the chemical fragmentation of the ablator are styrene, benzene, acetylene, carbon and hydrogen [12]. Both neutral and ionized forms have to be considered for each species in order to model the plasma formation. The following mixture is thus considered: $S, S^{+}, S^{2+}, A, A^{+}$, $A^{2+}, B, B^{+}, B^{2+}, H, H^{+}, C, C^{+}, C^{2+}, C^{3+}, C^{4+}, C^{5+}, C^{6+}$, where $S, A, B, H$ and $C$ correspond to styrene, acetylene, benzene, hydrogen and carbon, respectively. The density evolution of those species is related to the electron dynamics including photoionization, electron impact ionization, and electron recombination. Dissociations of styrene, benzene and acetylene molecules must also be described to model the chemical fragmentation of the ablator. The most relevant dissociations reactions are [12]:

$$
\begin{aligned}
& S \longrightarrow A+B \\
& S^{+} \longrightarrow A+B^{+} \\
& S^{2+} \longrightarrow \frac{1}{2} A+\frac{1}{2} A^{+}+\frac{1}{2} B^{+}+\frac{1}{2} B^{2+} \\
& A \longrightarrow 2 C+2 H \\
& A^{+} \longrightarrow C+C^{+}+2 H \\
& A^{2+} \longrightarrow 2 C^{+}+2 H \\
& B \longrightarrow 6 C+6 H \\
& B^{+} \longrightarrow 5 C+C^{+}+6 H \\
& B^{2+} \longrightarrow 4 C+2 C^{+}+6 H
\end{aligned}
$$

which take place when the ion-lattice temperature is about several $\mathrm{eV}$. The dynamics of each population is described as follows:

$$
\frac{\partial n_{X^{j}}}{\partial t}=\sum_{s} Q_{D, s}+Q_{I R}\left(X^{j-1} \leftrightarrow X^{j}\right)-Q_{I R}\left(X^{j} \leftrightarrow X^{j+1}\right)
$$

with $X \in\{S, A, B, H, C\}$ denoting the chemical species and $j \geq 0$ the ionization state. The first term corresponds to the dissociation rate where the sum is over all the reactions driving the evolution of the population $X$. For example, in the case of $B^{+}$, three dissociations rates have to be considered accounting for the reactions $(7 \mathrm{~b}),(7 \mathrm{c})$ and $(7 \mathrm{~h})$. The dissociation rate reads:

$$
Q_{D}=\beta n_{D} k_{a r r} \exp \left(-\frac{E_{a}}{k_{B} T_{i l}}\right)
$$

with $\beta$ the algebraic stoichiometric coefficient (negative for reactants, positive for products), $n_{D}$ the density of the molecule that dissociates, $k_{\text {arr }}$ the Arrhenius law prefactor, and $E_{a}$ the activation energy. We set $k_{a r r}=10^{13} \mathrm{~s}^{-1}$ corresponding to the characteristic time of dissociation of a molecule [23]. For the reactions given by Eqs. (7a)-(7c), one has $E_{a}=2.4 \mathrm{eV}$ and for the reactions given by Eqs. (7d)-(7i), $E_{a}=6 \mathrm{eV}$ [12]. The second and third term in Eq. (8] correspond to the transitions between the $j-1$-th and $j$-th ionization state, and the $j$-th and the $j+1$-th ionization state, respectively. It is assumed that these transitions can be due to photoionization (PI), electron impact ionization (EII) and electron recombination (ER) leading to :

$$
\begin{aligned}
Q_{I R}\left(X^{j-1} \leftrightarrow X^{j}\right) & =W_{P I}\left(X^{j-1} \rightarrow X^{j}\right) n_{X^{j-1}} \\
+W_{E I I}\left(X^{j-1}\right. & \left.\rightarrow X^{j}\right) n_{X^{j-1}}-W_{E R}\left(X^{j} \rightarrow X^{j-1}\right) n_{X^{j}}
\end{aligned}
$$

Photoionization of carbon and hydrogen atomic species is described within the Keldysh formalism for atoms [22]. In order to get a tractable model, this Keldysh expression derived for atoms is also used to calculate the photoionization rate of styrene, benzene and acetyelene, assuming the ionization energy is the main parameter driving the electron dynamics. Electron impact ionization is described with the Gryziński's theory [24] as a compromise between simplicity and accuracy. Concerning electron recombination, the approach of Zel'dovich [25] is adapted by substituting the Coulomb potential by the Debye-Hückel potential to account for screening effects which are significant at solid density:

$$
W_{E R}\left(X^{j} \rightarrow X^{j-1}\right)=\pi^{2} n_{e}^{2} j^{3} \sqrt{\frac{8 k_{B} T_{e}}{\pi m_{e}}}\left[\lambda_{D} W\left(\frac{2 e^{2}}{3 \lambda_{D} k_{B} T_{e}}\right)\right]^{5}
$$

where $\lambda_{D}=\sqrt{k_{B} T_{e} / 4 \pi n_{e} e^{2}}$ is the screening Debye length and $W$ is the principal branch of the $W$-Lambert function.

The evaluation of the density of each population with Eq. (8) allows to define the densities of electrons $n_{e}$, ions $n_{i}$ and neutrals $n_{n}$ :

$$
n_{e}=\sum_{X, j \geq 1} Z_{j} n_{X^{j}} ; n_{i}=\sum_{X, j \geq 1} n_{X^{j}} ; n_{n}=\sum_{X} n_{X}
$$

where $Z_{j}$ is the charge of the ion $n_{X^{j}}$, and the total density is given by $n_{T}=n_{i}+n_{n}$.

\section{Energy transfer}

When $T_{i l}<58 \mathrm{meV}$, the ablator is solid polystyrene and the energy transfer is mainly due to electron-phonon collisions. When $T_{i l}>58 \mathrm{meV}$, the ablator is constituted of molecules and atoms. In that case the energy transfer is mainly supported by electron-ion collisions.

For $T_{i l}<58 \mathrm{meV}$, as a first approximation, it is possible to assume that two optical phonons with energy $\hbar \omega_{L 0,1}=$ $33 \mathrm{meV}$ and $\hbar \omega_{L 0,2}=88 \mathrm{meV}$, and one acoustic phonon exist in polystyrene [12]. Thus, the energy transfer from electrons 
to phonons is given by :

$$
\left(\frac{\partial U_{e}}{\partial t}\right)_{\text {collisions }}=\left(\frac{\partial U_{e}}{\partial t}\right)_{A C}+\left(\frac{\partial U_{e}}{\partial t}\right)_{L O, 1}+\left(\frac{\partial U_{e}}{\partial t}\right)_{L O, 2}
$$

accounting for the different contributions of each phonon. The electron-acoustic phonon and electron-optical phonon energy transfer are respectively defined by :

$$
\begin{aligned}
& \left(\frac{\partial U_{e}}{\partial t}\right)_{A C}=v_{e t, a c} n_{e} k_{B}\left(T_{e}-T_{i l}\right) \\
& \left(\frac{\partial U_{e}}{\partial t}\right)_{L O}=v_{e t, o p} \hbar \omega_{L 0} n_{e}\left[g\left(T_{e}\right)-g\left(T_{i l}\right)\right]
\end{aligned}
$$

where $g(T)=\left[\exp \left(\hbar \omega_{L O} / k_{B} T\right)-1\right]^{-1}$ corresponds to the Bose-Einstein distribution and $v_{e t, a c}$ and $v_{e t, o p}$ are the energy transfer frequencies evaluated in Appendix $\mathrm{C}$.

For $T_{i l}>58 \mathrm{meV}$, the ablator is constituted of molecules and atoms. Because they are mostly ionized due to the laser, the energy transfer is mainly due to electron-ion interactions which is described by the energy transfer derived by Landau [26] :

$$
\left(\frac{\partial U_{e}}{\partial t}\right)_{\text {collisions }}=\frac{4 n_{e} n_{i} Z^{2} e^{4} \sqrt{2 \pi m_{e}} \ln \Lambda}{m_{e f f}\left(k_{B} T_{e}\right)^{3 / 2}} k_{B}\left(T_{e}-T_{i l}\right)
$$

where $m_{e f f}=\left(\sum_{X, j} m_{X^{j}} n_{X^{j}}\right) /\left(\sum_{j} n_{X^{j}}\right)$ is the effective mass of ions. It accounts for the variation of the mass of ions due to the dissociation of the molecular species. $\ln \Lambda$ corresponds to the Coulomb logarithm for which an approximation valid for dense plasmas is used [14]. Note that the energy transfer between electrons and neutral species is neglected. This is supported by the fact that neutrals are only present during a short period of time as shown in section III]

\section{RESULTS ON THE DYNAMICS OF THE SOLID-TO-PLASMA TRANSITION}

To perform numerical calculations with the model presented previously, a $351 \mathrm{~nm}$-laser is considered with a temporal Gaussian shape $I(t)=I_{0} \exp \left(-4 \ln 2\left(t-t_{\max }\right)^{2} / \tau^{2}\right)$ where $I_{0}, t_{\max }$ and $\tau$ are chosen such that they correspond to typical laser picket parameters used in ICF experiments [27]. This leads to $I_{0}=10^{14} \mathrm{~W} . \mathrm{cm}^{-2}, t_{\max }=200 \mathrm{ps}$ and $\tau=100 \mathrm{ps}$. The initial temperature for electrons and the ion-lattice system is set to $300 \mathrm{~K}$. Simulations begin when the laser intensity is high enough to quickly produce an electron density in the $\mathrm{CB}$ of the order of the initial electron density $\left(10^{10} \mathrm{~cm}^{-3}\right)$, i.e. when the laser beam modifies significantly the material. Calculations are stopped when the electron density remains constant, ensuring the solid-to-plasma transition is ended since no more electrons can be generated. It then turns out that simulations are performed between $-75 \mathrm{ps}$ and $100 \mathrm{ps}$. The laser is already intense enough for $t<0 \mathrm{ps}$ to create a significant free electron density, and the electron density remains constant after $t>100 \mathrm{ps}$.
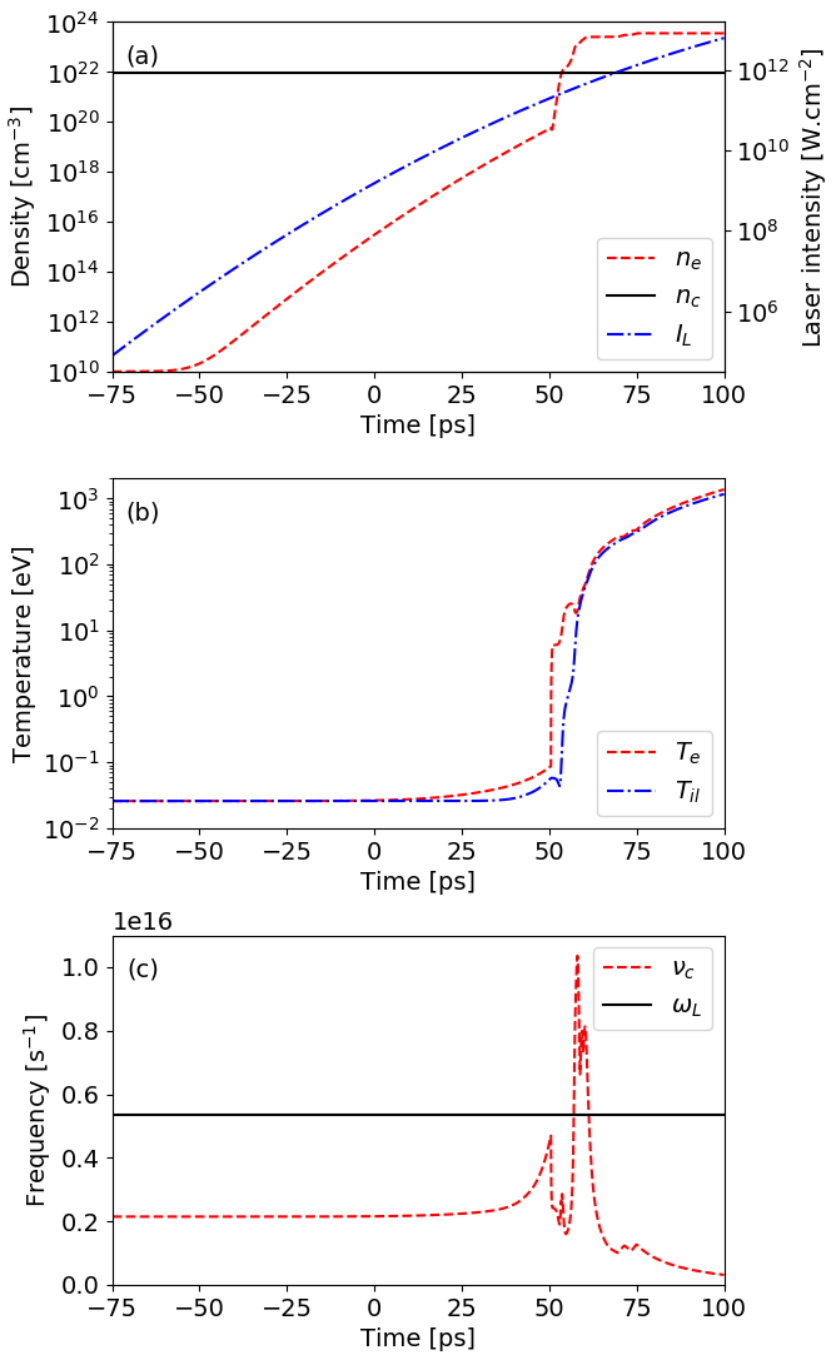

FIG. 1. Evolution of (a) the electron density $n_{e}$ and the laser intensity $I_{L}$, (b) electron temperature $T_{e}$ and ion-lattice temperature $T_{i l}$ and (c) the electron collision frequency $v_{c}$ as a function of time. The critical density $n_{c}=m_{e} \omega_{L}^{2} / 4 \pi e^{2}$ and the laser frequency $\omega_{L}$ are given for purpose of comparison. A Gaussian profile is considered for the laser intensity with a maximum of $10^{14} . \mathrm{Wcm}^{-2}$ reached at $200 \mathrm{ps}$ and a duration of $100 \mathrm{ps}$.

Figure 1 shows the temporal evolution of the electron density, electron and ion-lattice temperatures, and the electron collision frequency between -75 ps and 100 ps.

Regarding the electron density, a monotonic behavior is observed when $t<50 \mathrm{ps}$. The ablator is solid polystyrene, this variation of the electron density is driven by the photoionization process. The electron impact ionization has a weak influence because the laser is not intense enough to promote a high number of electrons to the third level and initialize the electron breakdown. For $t>50 \mathrm{ps}$, the ablator is constituted of molecules and atoms and the electron generation is driven by electron impact ionization. The ionization energies of the species constituting the ablator are about $8 \mathrm{eV}$ 
for the lowest one, simultaneous absorption of three photons is required to photoionize which makes this rate low. Since the electron temperature is higher than $10 \mathrm{eV}$ for $t>50 \mathrm{ps,}$ the impact ionization process becomes dominant. The first increase of the electron density going from $n_{e} \approx 6 \times 10^{19} \mathrm{~cm}^{-3}$ to $n_{e} \approx 10^{22} \mathrm{~cm}^{-3}$ corresponds to the ionization of styrene molecules since the ion-lattice system remains cold and only styrene molecules are present. The second increase where the electron density goes from $n_{e} \approx 10^{22} \mathrm{~cm}^{-3}$ to $n_{e} \approx 2.5 \times 10^{23}$ $\mathrm{cm}^{-3}$ correspond to the simultaneous ionizations of benzene, acetylene, hydrogen and carbon atomic species. The ionlattice temperature increases from $0.1 \mathrm{eV}$ to $10 \mathrm{eV}$ and all the dissociation reactions given by Eq. (7a)-(7i) take place. Finally, the last two increases of the electron density taking place between 70 ps and 75 ps are associated to the fifth and sixth ionization of atomic carbon. The ion-lattice temperature is about a few hundreds of $\mathrm{eV}$ and the ablator is mostly composed of hydrogen and carbon atomic species. When $n_{e} \approx 3.5 \times 10^{23} \mathrm{~cm}^{-3}$, all the species are fully ionized, no more electrons can be generated and the electron density remains constant. Thus, the initial solid ablator has transformed into a fully ionized plasma, and the solid-to-plasma transition can be considered as finished. Note that as long as $t<54 \mathrm{ps}$, the electron density is smaller than the critical density. For these early times where the intensity is lower than a few $10^{11} \mathrm{~W} \cdot \mathrm{cm}^{-2}$, the laser beam can propagate throughout the ablator and be absorbed in the fuel area [28]. This is the shinethrough issue which may reduce the target efficiency [5, 29].

Concerning the temperatures and the electron collision frequency, two behaviors associated to $t<50 \mathrm{ps}$ and $t>50$ ps can be noticed, as for the electron density evolution. For $t<50 \mathrm{ps}$, electrons remains relatively cold, the electron temperature does not exceed $0.1 \mathrm{eV}$. Although the electron collision is important, $v_{c} \approx 2 \times 10^{15} \mathrm{~s}^{-1}$, leading to an efficient laser heating, the energy transfer from electrons to the lattice is also important and the whole laser energy acquired by the electrons is directly transferred to the lattice. This results in a slow increase of the electron temperature. However, even if the internal energy of the lattice increases due to the electronphonon energy transfer, it does not lead to a significant heating of the lattice due to the large value of the polystyrene heat capacity.

For $t \approx 50 \mathrm{ps}$, the electron temperature increases rapidly due to the drop of the energy transfer. At this moment where $T_{i l} \approx 58 \mathrm{meV}$, the chemical constitution of the ablator changes going from solid polystyrene to styrene which is a molecular specie. The nature of the energy transfer is modified because collisions are no longer between electrons and phonons but between electrons and ions. This drop of the energy transfer leads to a heating of the ions much less efficient and the ion-lattice temperature decreases because, on the other hand, the internal energy of styrene is modified due to ionization. Note that a signature of the phase transition is the drop of the electron collision frequency at $t \approx 50 \mathrm{ps}$.

For $t>50 \mathrm{ps}$, the electron temperature and the ion-lattice temperature increase due to the laser heating and electronion energy transfer, respectively. Regarding the behavior of the electron collision frequency, it first increases because the electron density increases due to ionizaition while the electron temperature remains relatively constant around $10 \mathrm{eV}$. Then, the electron collision frequency decreases because the electron density does not increase much going from $2.5 \times 10^{23}$ $\mathrm{cm}^{-3}$ to $3.5 \times 10^{23} \mathrm{~cm}^{-3}$ while the electron temperature increase significantly.

In order to have a closer look at the evolution of the chemical composition of the ablator in the plasma state, Fig 2 shows the temporal evolution of the density of styrene, acetylene, benzene molecules, and the density of hydrogen and carbon atomic species during the solid-to-plasma transition. They are shown between $t \approx 50 \mathrm{ps}$ (moment when polystyrene dissociates into styrene molecules) and $t \approx 60 \mathrm{ps}$ (densities of benzene and acetylene molecules are small enough so that only carbon and hydrogen atomic species remain). Such a temporal window ensures to capture the entire chemical fragmentation of the ablator.

Because the ion-lattice temperature increases during the solid-to-plasma transition, the ablator is successively constituted of polystyrene, styrene molecules, benzene and acetylene molecules, and finally carbon and hydrogen atomic species. This chemical fragmentation can be observed with the increase of the total density on a timescale of $\sim 10 \mathrm{ps}$ which is not negligible compared with the whole solid-toplasma transition timescale of $\sim 100 \mathrm{ps}[5]$. Thus the chemical fragmentation of the ablator cannot be neglected in the description of the solid-to-plasma transition. However, benzene and acetylene are only present during a few picoseconds. This is due to the fact that the ion-lattice temperature increases very quickly from $0.1 \mathrm{eV}$ to several tens of $\mathrm{eV}$. Thus, as a possible increase in computational efficiency for purposes of implementation in a hydrocode, it could be assumed that styrene dissociates directly into carbon and hydrogen atomic species.

Regarding the temporal evolution of neutral and ionized species, neutrals exist only during a very short period of time compared to the solid-to-plasma transition timescale. Just after the polystyrene dissociation, the ablator is mainly composed of neutrals molecules. Because the electron temperature is small at this moment, there is no impact ionization. The latter process starts when electrons are hot enough, then neutral and ion densities decreases and increases, respectively. After a fast decrease between $t=52 \mathrm{ps}$ and $t=54$ ps because of the ionization of styrene molecules, the neutral density increases between $t=54 \mathrm{ps}$ and $t=58 \mathrm{ps}$. This is due to the chemical fragmentation of the ablator which leads to the formation of new neutral species. Regarding chemical reactions given by Eqs. (7a)-(7i), it can be observed that neutral species are created during the dissociation of ions. Finally, the neutral density decreases again for $\mathrm{t}>58$ ps because the chemical fragmentation is ended. Neutrals species do no longer appear and already present neutrals disappear because they are ionized by electron impact. Even though neutrals are present during a short period of time, considering them enable not to have to use some tweaks to properly describe the solid state transition to a mixture of ionic species. This approach ensures the continuity of the quantities such as the electron collision frequency. 

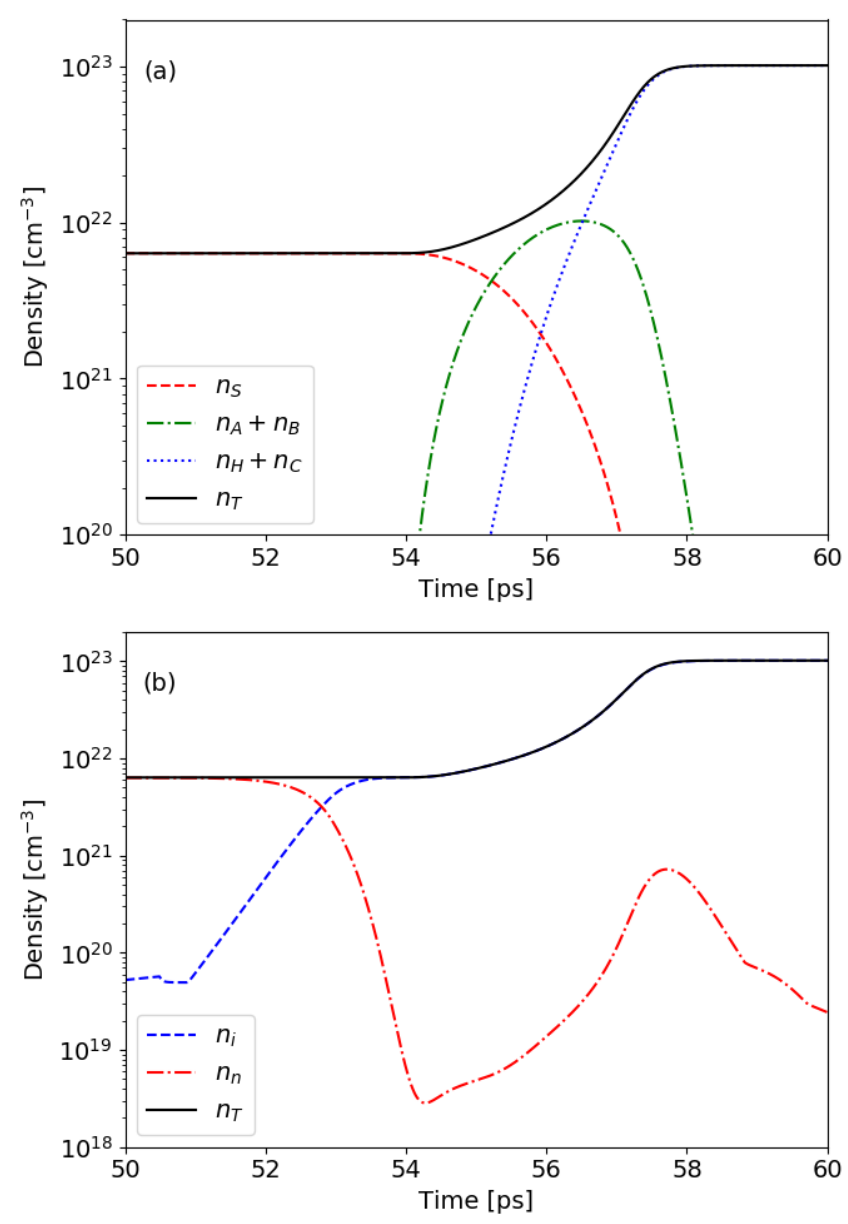

FIG. 2. (a) Temporal evolution of the density of styrene molecules $n_{S}$, acetylene molecules $n_{A}$, benzene molecules $n_{B}$, hydrogen atoms $n_{H}$, carbon atoms $n_{C}$, and the total density $n_{T}$. The evolution of $n_{A}$ and $n_{B}$ as well as $n_{H}$ and $n_{C}$ are plotted together because they appear and disappear for the same ion-lattice temperature (See Section IIC2 2. For each specie, the density corresponds to the density of neutrals plus the density of ions. (b) Temporal evolution of the total ion density $n_{i}$, total neutral density $n_{n}$ and the total density $n_{T}=n_{i}+n_{n}$. As a reminder, the total ion and neutral densities $n_{i}$ and $n_{n}$ are defined by Eq. (12). The same laser intensity profile as the one used for Fig. 1 is considered.

The solid-to-plasma transition timescale as predicted by this improved model is now addressed. Figure 3 shows the temporal evolution of the electron density and both electron and ion-lattice temperatures for three maximum laser intensities $I_{0}$ commonly used in ICF experiments [27]. The solid-toplasma transition timescale is defined as the duration necessary to fulfill two criteria: $n_{e} \geq n_{c}$ and $T_{i l} \geq 0.1 \mathrm{eV}$. The second criterion ensures the periodicity of the lattice is broken (plasmas in general are disordered matter). Figure 3 shows that the temporal shape of $n_{e}, T_{e}$ and $T_{i l}$ during the solid-toplasma transition does not depend on the maximum laser intensity, it mainly consists of a temporal shift. However, the laser intensity has a slight influence on the solid-to-plasma
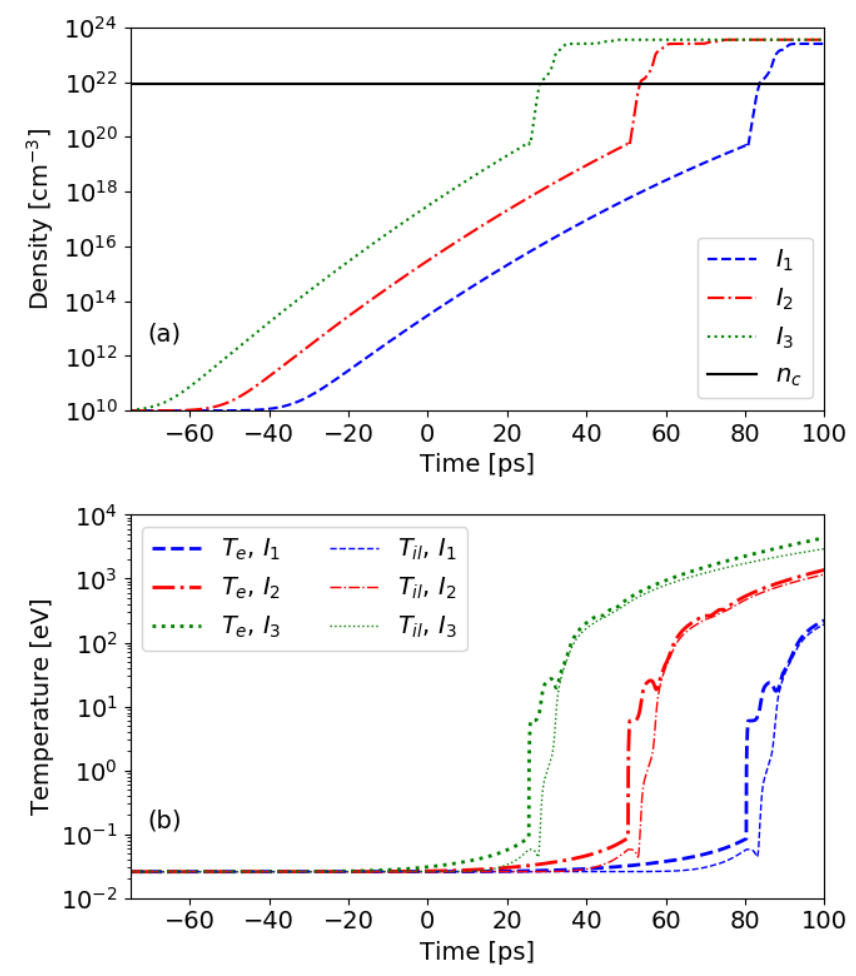

FIG. 3. (a) Evolution of the electron density as a function of time. (b) Evolution of the electron temperature $T_{e}$ (thick lines) and the ionlattice temperature $T_{i l}$ (thin lines) as a function of time. Simulations are performed with a Gaussian laser shape with a duration of $100 \mathrm{ps}$ for the three following maximum laser intensities $I_{1}=10^{13} \mathrm{~W} \cdot \mathrm{cm}^{-2}$, $I_{2}=10^{14} \mathrm{~W} . \mathrm{cm}^{-2}$ and $I_{3}=10^{15} \mathrm{~W} . \mathrm{cm}^{-2}$ reached at $200 \mathrm{ps}$. For the temperatures, solid and dashed lines corresponds to electron and ionlattice temperatures respectively. The critical density $n_{c}$ is shown for purpose of comparison.

\begin{tabular}{|c|c|c|c|}
\hline Maximum laser intensity $\left[\mathrm{W} . \mathrm{cm}^{-2}\right]$ & $10^{13}$ & $10^{14}$ & $10^{15}$ \\
\hline Solid-to-plasma transition timescale [ps] & 125 & 110 & 100 \\
\hline
\end{tabular}

TABLE I. Solid-to-plasma transition timescale as a function of the maximum laser intensity

transition timescale as summarized in Table M More intense is the laser, more energy is absorbed by the ablator, and faster the plasma state is reached. The presently obtained timescale is consistent with previsous estimations providing $\sim 100 \mathrm{ps}$ [5]. Indeed the ablator remains a relatively long time in the solid state before undergoing any phase transition, the electron dynamics is mainly driven by photoionization of polystyrene. Thus, electron impact ionization taking place when the ablator is constituted of molecules and atomic species has only a weak influence on the solid-to-plasma transition timescale. 


\section{COMPARISON TO THE INITIAL PLASMA CONDITIONS ASSUMED IN CURRENT HYDROCODES}

The model introduced in this work is now compared with initial conditions corresponding to a fully ionized carbonhydrogen plasma. These are standard initial conditions used in ICF hydrocodes where in particular the electron density is over critical. The present model is also compared with the one introduced by Duchateau et al. [5]. For the three models, the initial temperature of electrons and ions is $300 \mathrm{~K}$ and a Gaussian laser pulse with the same parameters as previously is used. Figure 4 provides the temporal evolution of the electron density, the electron temperature and the ion-lattice temperature as predicted by the three approaches.

Regarding the electron density, the model presented in this work and the one developed by Duchateau et al. give similar results as long as $t<50 \mathrm{ps}$, i.e. when solid polystyrene still stands, because the same description of the electron dynamics (photoionization with band structure) is used in both models. For $t>50 \mathrm{ps}$ where chemical fragmentation takes place, the disagreement is due to the fact that Duchateau et al. still assume the same model for electron dynamics as previously whereas impact ionization of molecules and atoms becomes significant. Since the present improved model accounts for full ionization, the electron density of the initial plasma model $\left(\sim 3.5 \times 10^{23} \mathrm{~cm}^{-3}\right)$ is retrieved.

Considering electron and ion-lattice temperatures at the end of the solid-to-plasma transition, similar results are obtained from our improved model and from the case where the ablator is assumed to be initially a fully ionized plasma. Because the laser absorption by the ablator leading to the electron generation and the chemical fragmentation of the ablator takes place for laser intensities much smaller than those associated to the heating of the electrons and the ion-lattice system when the ablator is totally ionized, the total laser energy absorbed by the ablator is about the same whether the solid-to-plasma transition is considered or not. However the initially fully ionized plasma leads to an ablator heated earlier since the electron density is much higher at the beginning of the interaction, which makes more efficient the laser heating of electrons and the electron-ion energy transfer. We thus expect that the ablator expansion takes place later when the solid-to-plasma transition is considered. Compared with the first attempt of model of solid-to-plasma transition, the interest of the present model is clearly shown by the differences in electron and ion temperatures. This discrepancy is due to improved model of collision frequencies and chemical fragmentation affecting the electron dynamics.

Compared with an initial plasma state, previous results have shown that accounting for the solid-to-plasma transition is expected to modify electron density and transient temperatures which drive both the laser dynamics and shock wave formation. Such conclusions were drawn assuming no hydrodynamics. This assumption can now be evaluated a posteriori to confirm previous predictions. For this purpose, the ablator expansion due to laser heating, $\delta x$, is estimated by integrating the ion sound speed $c_{s}$ over the time $\delta x=\int_{t_{0}}^{t} c_{s}(\tau) \mathrm{d} \tau$ where the plasma neutrality is assumed. $t_{0}=-75$ ps corresponds to
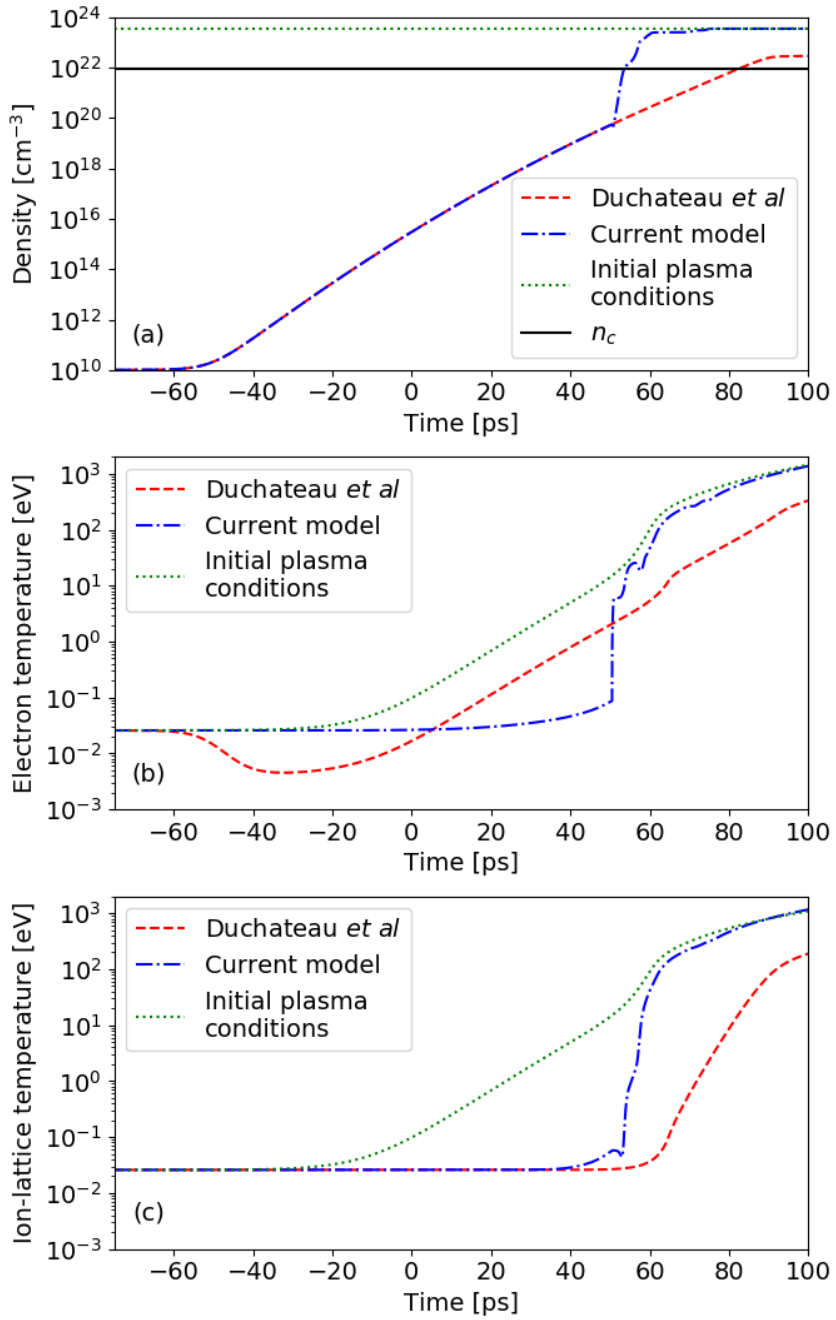

FIG. 4. Electron density (a), electron temperature (b) and ionlattice temperature (c) during the solid-to-plasma evaluated for different models. The red curve corresponds to the model developed by Duchateau et al [5]. The blue curve corresponds to the model presented in this work. The green curve corresponds to initial conditions that can be encountered in current hydrocodes, i.e. the ablator is assumed to be initially a fully ionized carbon-hydrogen plasma. The same laser intensity profile as the one used in Sec. III is considered.

the initial time and the sound speed is [30]:

$$
c_{s}=\sqrt{\frac{\gamma_{e} Z k_{B} T_{e}+\gamma_{i} k_{B} T_{i l}}{m_{i}}}
$$

where $\gamma_{e}=1$ and $\gamma_{i}=3$ are the electron and ion heat capacity ratios, respectively [31]. The evolution of the ablator expansion during the solid-to-plasma transition is shown in Fig. 5 ,

Two main behaviors can be observed as for the electron density, electron temperature and ion-lattice (see Fig. 11. If $t<50 \mathrm{ps}$, the ion-lattice system remains cold and the ablator expansion is negligible (less then $0.1 \mu \mathrm{m}$ ). If $t>50 \mathrm{ps}$, the ion-lattice system is heated very quickly and reaches high 


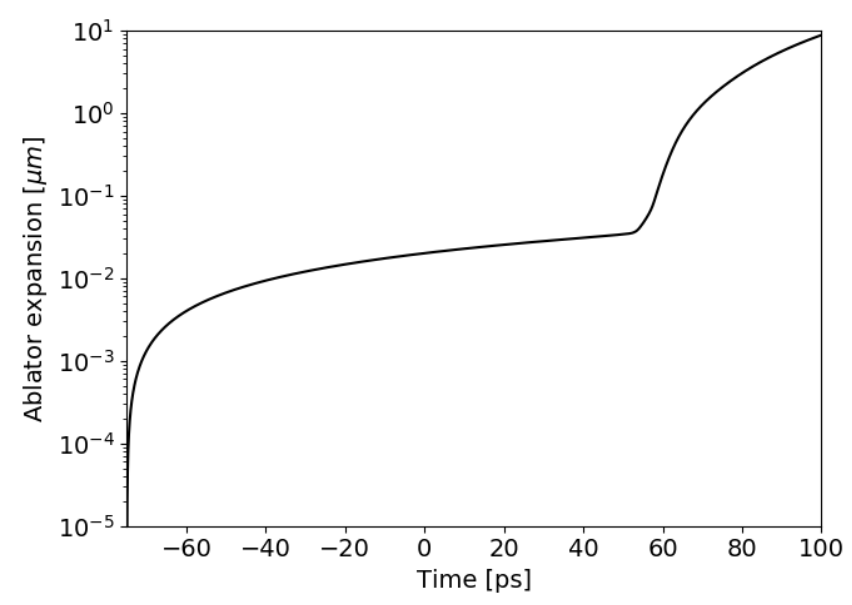

FIG. 5. Ablator expansion during the solid-to-plasma transition. The same laser intensity profile as the one used in Sec. III is considered.

temperatures in excess of several hundreds of eV. The ablator expansion, which may be of several $\mu \mathrm{m}$, then has to be considered. Polystyrene ablators typically used in ICF experiments have a thickness of several tens of $\mu \mathrm{m}[32-34]$. For $t \sim 70$ ps where the solid-to-plasma transition is mainly finished, the expansion is of the order of $1 \mu \mathrm{m}$, i.e. less than $10 \%$ of the ablator thickness. We thus confirm that hydrodynamics can be neglected during the solid-to-plasma transition. However the solid-to-plasma transition may have an influence on the subsequent hydrodynamics as suggested in recent experiments in order to explain differences on the shock velocities modulations between experimental and numerical results [11]. This discrepancy may be explained by the evolution of the matter pressure with the laser intensity which scales differently depending on the state of matter, thus forming different gradients responsible for shock formation and propagation.

\section{SCALING LAW OF MATTER PRESSURE WITH RESPECT TO LASER INTENSITY}

In above mentioned experiments [11], shock velocities modulations induced in a polystyrene foil by a laser beam including speckles (spatial intensity modulations) were measured with a 2D VISAR to study the laser imprint. By using a state-of-the-art hydrocode assuming an initial plasma state, a good agreement of numerical simulations with experimental observations was obtained by increasing the intensity modulations by a factor of two. The later modification was supported by invoking the role of the initial solid state. By considering a fully ionized plasma, the relative pressure exerted by a beam profile, $\delta P / P$, is equal to the relative intensity variations $\delta I / I$. An ideal gas EOS is assumed with a constant density and a temperature resulting from linear laser absorption. Considering the initial solid state, absorption becomes non linear (ionization through two-photon absorption) and free electron density depends on laser intensity. Then $\delta P / P=\alpha \delta I / I$, or
$P \propto I^{\alpha}$, where the coefficient $\alpha$ accounts for the plasma formation. Compared with an initial fully ionized plasma state, the solid-to-plasma transition is thus expected to modify the scaling law of the pression as a function of the laser intensity. This section is devoted to the investigation of the modification of the previous scaling law exponent due to the solid-toplasma transition.

First, the scaling law exponent is evaluated for a fully ionized plasma. The obtained value will be a reference to check the predictions of the present solid-to-plasma model. The total pressure $p$ is given by considering electron and ion contributions, $p=p_{e}+p_{i}$, with $p_{e}$ and $p_{i}$ the electron and ion pressures, respectively. Assuming electrons and ions have the same temperature (long enough time) and ideal gas EOS, the total pressure reads:

$$
p=\left(1+\frac{1}{Z}\right) n_{e} k_{B} T_{e}
$$

with $Z=n_{e} / n_{i}$ the averaged ionization degree of the plasma. Thus, the scaling law of the pressure as a function of the laser intensity is due to the evolutions of both electron density $n_{e}$ and electron temperature $T_{e}$. For a fully ionized plasma where $n_{e}$ is constant, only the electron temperature scaling needs to be evaluated. This is done by Eq. 1 which, after simplification, reads:

$$
\frac{\partial T_{e}}{\partial t}=\frac{16 \pi e^{2} v_{c}}{3 c k_{B} m_{e} \omega_{L}^{2}} I_{L}(t)
$$

with $c$ the light velocity. $\quad v_{c} \ll \omega_{L}$ is assumed for a hot enough plasma. Thus, the scaling law of the temperature is obtained owing to the scaling law of the electron collision frequency. The assumption of a hot plasma enables to write $v_{c} \propto T_{e}^{-3 / 2} \ln \Lambda$. Neglecting the electron temperature dependency of the Coulomb logarithm, the previous equation can be solved leading to:

$$
T_{e}^{5 / 2}-T_{0}^{5 / 2} \propto I_{0} F(t)
$$

with $T_{0}$ the initial electron temperature and $F(t)=\int_{-\infty}^{t} f(t) \mathrm{d} t$ where the laser intensity profile is $I_{L}(t)=I_{0} f(t)$ where $f(t)$ is the temporal profile. With $T_{0} \ll T_{e}$, the scaling law of the pressure as a function of the laser intensity for a fully ionized plasma reads:

$$
p \propto I_{0}^{2 / 5}
$$

In order to study the modification of this scaling law due to the solid-to-plasma transition, simulations were performed by using the model presented in Sec. [I] and a Gaussian shape for the temporal laser intensity profile with the same parameters as those used in Sec. III. Simulations were performed for different maximum laser intensities $I_{0}$ ranging from $10^{12}$ W.cm ${ }^{-2}$ to $10^{15} \mathrm{~W} . \mathrm{cm}^{-2}$. Figure 6 shows the evolution of the total pressure and the electron density as a function of time and of the laser intensity.

It can be observed on Fig. 6(b) that the pressure evolution exhibits four behaviors. The first three behaviors are related 

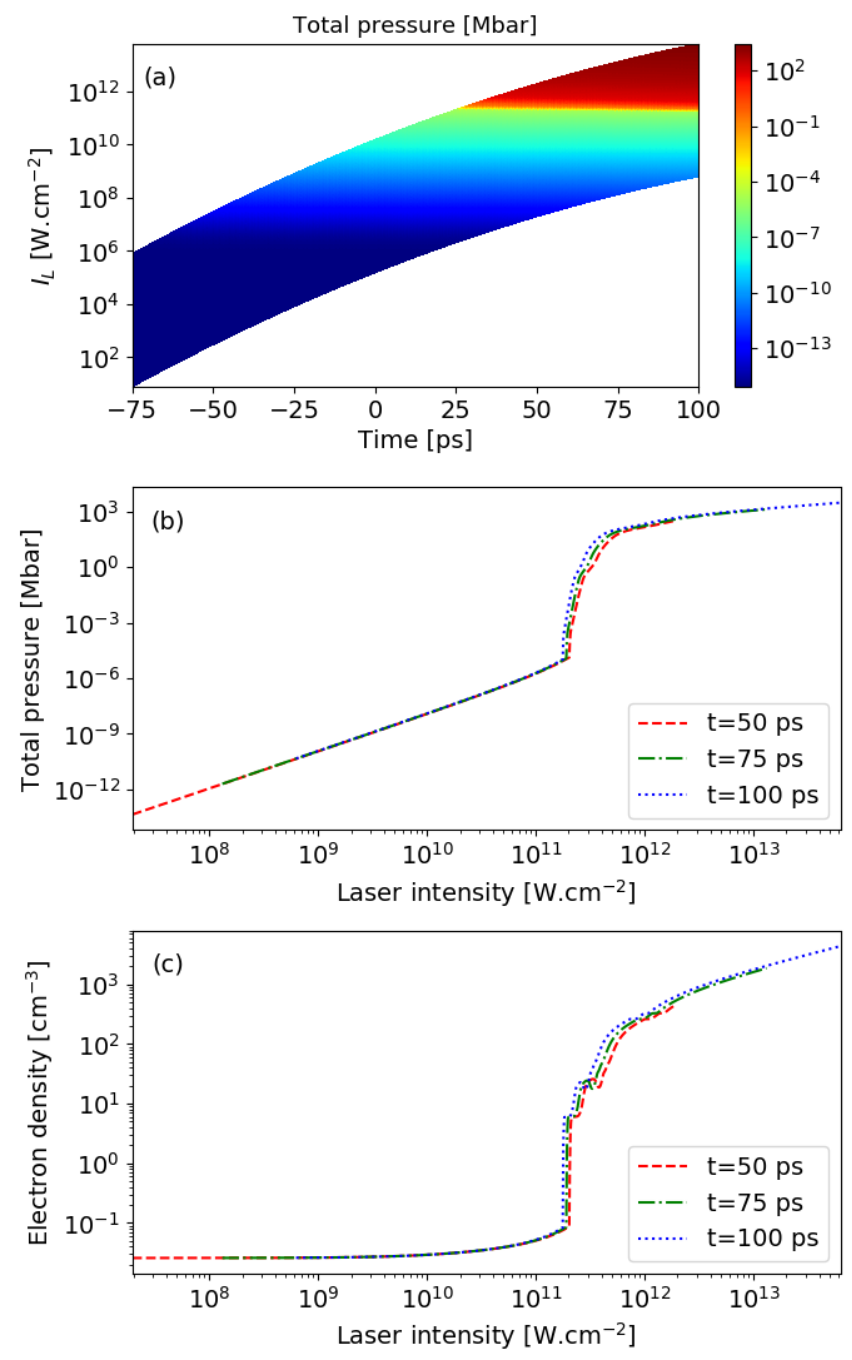

FIG. 6. (a) Total pressure as a function of time and of the laser intensity, (b) total pressure as a function of the laser intensity for three different instants, (c) electron density as a function of the laser intensity for three different instants.

to the evolution of the electron density while the last one is related to the evolution of the electron temperature. When $I_{L}<2.0 \times 10^{11} \mathrm{~W} . \mathrm{cm}^{-2}$, the ablator is solid polystyrene and the electron density scales as $n_{e} \propto I_{L}^{2}$ as shown by Fig. 6.c). This scaling law is also valid for the pressure, because the electron temperature remains relatively constant for the solid polystyrene state (see Fig 1(b)). In that case the scaling of the pressure is given by the scaling of the electron density. Thus, when $I_{L}<2.0 \times 10^{11} \mathrm{~W} . \mathrm{cm}^{-2}$, the pressure scales as $p \propto I_{L}^{2}$. For laser intensities between $2.0 \times 10^{11} \mathrm{~W} . \mathrm{cm}^{-2}$ and $5.0 \times 10^{11} \mathrm{~W} . \mathrm{cm}^{-2}$, the pressure exhibits a fast increase which is due to the increase in the electron density induced by dissociations and ionizations of all molecular and atomic species present at this moment. A rough fit of the pressure leads to the following scaling law: $p_{e} \propto I_{L}^{11}$. For laser intensities between $6.0 \times 10^{11} \mathrm{~W} . \mathrm{cm}^{-2}$ and $2.0 \times 10^{12} \mathrm{~W} . \mathrm{cm}^{-2}$, the evolution of the pressure is due to the increase of the electron density going from $2.5 \times 10^{23} \mathrm{~cm}^{-3}$ to $3.5 \times 10^{23} \mathrm{~cm}^{-3}$. This evolution is due to the last two ionizations of carbon taking place for $I_{L} \approx 10^{12} \mathrm{~W} \cdot \mathrm{cm}^{-2}$. A numerical fit leads to $p \propto I_{L}^{1.2}$. Finally, for $I_{L}>3.0 \times 10^{12} \mathrm{~W} . \mathrm{cm}^{-2}$, the electron density remains constant and the evolution of the pressure is due to variations of electron temperature. In that case, a fitting of the pressure leads to $p \propto I_{L}^{0.5}$. This scaling law is similar to the one given by Eq. 21. The exponent difference ( 0.5 vs 0.4$)$ is due to the dependence of the collision frequency on the electron temperature. Eq. 21 has been obtained assuming a constant Coulomb logarithm, neglecting the electron-ion energy transfer, and assuming $v_{c} \ll \omega_{L}$ to evaluate the energy absorption, the last two assumptions leading to Eq. 19 .

These results show that the solid-to-plasma transition strongly modifies the scaling law of the pressure as a function of the laser intensity due to the free electron generation. By fitting the curves over a relevant intensity range, the average scaling law exponent is of the order of 3 . This work thus confirms a posteriori the approach discussed in [11] to account for experimental observations. Note that a similar experimental study has been carried out recently with different parameters [35] where the photoionization of the solid target is due to one-photon absorption. As in [11], state-of-the-art numerical simulations with an initial plasma state cannot account accurately of experimental observations. Since the primary ionization process of the solid plastic is due to a linear absorption process, the authors of [35] concluded that the introduction of the solid-to-plasma transition is not enough to explain discrepancies. Here we have shown that the scaling law exponent value is mainly due to variations of the free electron density, regardless the exact ionization process (of course the precise value of the exponent depends on the ionization processes). The solid-to-plasma transition may thus also mainly explain experimental and theoretical discrepancies observed in [35].

\section{CONCLUSION AND OUTLOOKS}

An improved description of the solid-to-plasma transition has been proposed in this work enabling to investigate in particular the temporal evolution of the electron density, electron temperature and ion-lattice temperature during the early interaction between the ablator and typical laser pickets used in ICF experiments. They exhibit two distinct behaviors associated to the chemical composition of the ablator. As long as the ion-lattice temperature is smaller than $58 \mathrm{meV}$, the ablator is in the solid polystyrene state. The electron density is driven by the laser-induced photoionization and the electron and ion-lattice temperatures are driven by collisions between electrons and phonons. For higher temperatures, polystyrene dissociates, transforming into various molecular and atomic species. The electron density is then driven by electron impact ionization while the heating of the electrons and of the ionlattice system is driven by electron-ion collisions. This model leads to a non negligible solid-to-plasma transition timescale of about $100 \mathrm{ps}$, which is about the order of magnitude of laser pickets duration. The present model can estimate the 
density of carbon and hydrogen ions at the end of the solidto-plasma transition, as well as electron and ion temperatures, which makes it suitable for its implementation in an ICF hydrocode.

The chemical evolution of the ablator going from polystyrene to a mixture of carbon and hydrogen atomic species has been investigated. It takes place over a period of time of about $10 \mathrm{ps}$, which is not negligible compared with the solid-to-plasma transition timescale. However, some intermediate chemical species are present only during a few ps and should be neglected for application purposes. By extrapolating the scenario of polystyrene fragmentation, a general scenario of fragmentation of plastics can be deduced. Indeed, in the ICF framework, and more generally within the laserplasma interaction context, polystyrene is only one among a large number of plastics that can be used, in particular of the case of complex target coating or the use of foams [3638]. We thus propose a simpler two-step scenario for the plasma formation: (i) The plastic (polymer) dissociates into monomers around the ceiling temperature; (ii) monomers dissociate into atomic species when the ion-lattice temperature is in between 3 and $8 \mathrm{eV}$ which corresponds to the typical range of chemical binding energies.

Finally, by investigating the evolution of the matter pressure as a function of the laser intensity, we have shown that the strong modification of the scaling law exponent is due to the solid-to-plasma transition where the free electron density varies. The solid-to-plasma transition is thus expected to have a significant influence on the shock formation and propagation for the laser imprint issue, and on the shinethrough issue as already demonstrated with a simplified model [28]. To definitely confirm these expectations, the model presented in this work will be implemented in a state-of-the-art multidimensional radiative hydrodynamic code. By doing so, it will be possible in addition to determine whether the early-time evolution of the ablator has a significant influence on the target implosion and performance such as the neutral yield within direct-drive ICF conditions.

\section{ACKNOWLEDGMENTS}

The authors acknowledge Vladimir Tikhonchuk for his fruitful comments about this work. CEA is also acknowledged for supporting these studies. SXH acknowledges the support by the US Department of Energy National Nuclear Security Administration under Award No. DE-NA0003856, the University of Rochester, and the New York State Energy Research and Development Authority.

\section{Appendix A: Two-temperature model with a non-constant number of particles}

Let us consider a thermodynamic system with a dependence on the number $N$ of particles. Thus, the total differential of the energy $U$ of the system reads [39]:

$$
\mathrm{d} U=T \mathrm{~d} S-P \mathrm{~d} V+\mu \mathrm{d} N
$$

where $T, S, P, V$ and $\mu$ are the temperature, entropy, pressure, volume and chemical potential, respectively. This equation leads to the definition of $P$ and $\mu$ :

$$
P=-\left(\frac{\partial U}{\partial V}\right)_{S, N} \quad ; \quad \mu=\left(\frac{\partial U}{\partial N}\right)_{S, V}
$$

By using the free energy $F$ defined by $F=U-T S$, which its total differential is $\mathrm{d} F=-S \mathrm{~d} T-P \mathrm{~d} V+\mu \mathrm{d} N$, the entropy can be defined as $S=-(\partial F / \partial T)_{V, N}$. Because $F$ is a function of $T, V$ and $N$, the entropy is a function of these same variables and its total differential is:

$$
\mathrm{d} S=\left(\frac{\partial S}{\partial T}\right)_{V, N} \mathrm{~d} T+\left(\frac{\partial S}{\partial V}\right)_{T, N} \mathrm{~d} V+\left(\frac{\partial S}{\partial N}\right)_{T, V} \mathrm{~d} N
$$

By substituting this expression for the differential of the entropy in Eq. A1, this allows to write the total derivative of the energy as:

$$
\mathrm{d} U=C_{v} \mathrm{~d} T-\tilde{p} \mathrm{~d} V+\tilde{\mu} \mathrm{d} N
$$

with $C_{v}=T(\partial S / \partial T)_{V, N}$ the heat capacity of a system with a variable number of particles as defined by Landau [39]. The partial derivatives over $V$ and $N$ have been gathered to defined an effective pressure $\tilde{p}$ and an effective chemical potential $\tilde{\mu}$ as follows:

$$
\begin{aligned}
& \tilde{p}=-\left(\frac{\partial U}{\partial V}\right)_{T, N}=P+T\left(\frac{\partial S}{\partial V}\right)_{T, N} \\
& \tilde{\mu}=\left(\frac{\partial U}{\partial N}\right)_{T, V}=\mu+T\left(\frac{\partial S}{\partial N}\right)_{T, V}
\end{aligned}
$$

Finally, the variations of internal energy is given by:

$$
\frac{\partial U}{\partial t}=C_{v} \frac{\partial T}{\partial t}-\tilde{p} \frac{\partial V}{\partial t}+\tilde{\mu} \frac{\partial N}{\partial t}
$$

\section{Appendix B: Thermodynamic quantities in the free electron gas} approximation

The two electron thermodynamic quantities of interest in the two-temperature model are the electron heat capacity $C_{e}$ and the effective chemical potential $\mu_{e f f}$. As the ablator does not significantly expand during its solid-to-plasma transition, the electron density can be very large. Thus, it is assumed electrons can be degenerated and the free electron gas approximation is used to recover both degenerate and non-degenerate regime. Thus, the electron energy reads:

$$
U_{e}=\frac{\sqrt{2} m_{e}^{3 / 2}}{\pi^{2} \hbar^{3}} \int_{0}^{+\infty} \frac{\varepsilon^{3 / 2}}{e^{\left(\varepsilon-\mu_{e}\right) / k_{B} T_{e}}+1} \mathrm{~d} \varepsilon
$$

which can be written in terms of the complete Fermi-Dirac integral:

$$
U_{e}=\frac{\sqrt{2} m_{e}^{3 / 2}}{\pi^{2} \hbar^{3}}\left(k_{B} T_{e}\right)^{5 / 2} F_{3 / 2}\left(\frac{\mu_{e}}{k_{B} T_{e}}\right)
$$


According to Landau \& Lifshitz [39], the heat capacity of a system with a variable number of particles is defined by $C_{v}=$ $T(\partial S / \partial T)_{V, N}$. By using Eq. (A1) and the fact that $T, V$ and $N$ are independent variables, this allows to define $C_{v}$ in terms of $U_{e}$ :

$$
C_{v}=\left(\frac{\partial U_{e}}{\partial T}\right)_{V, N}
$$

Thus, by using Eq. $\overline{\mathrm{B} 2}$, this leads to:

$$
\begin{aligned}
C_{v} & =\frac{\sqrt{2} m_{e}^{3 / 2}}{\pi^{2} \hbar^{3}}\left[\frac{5}{2} k_{B}^{5 / 2} T_{e}^{3 / 2} F_{3 / 2}\left(\frac{\mu_{e}}{k_{B} T_{e}}\right)\right. \\
& \left.+\frac{3}{2}\left(k_{B} T_{e}\right)^{5 / 2} F_{1 / 2}\left(\frac{\mu}{k_{B} T_{e}}\right) \frac{\partial}{\partial T_{e}}\left(\frac{\mu_{e}}{k_{B} T_{e}}\right)\right]
\end{aligned}
$$

where the property of the Fermi-Dirac integral $\mathrm{d} F_{j}(x) / \mathrm{d} x=$ $j F_{j-1}(x)$ has been used. Finally, as the electron density is defined by:

$$
n_{e}=\frac{\sqrt{2} m_{e}^{3 / 2}}{\pi^{2} \hbar^{3}}\left(k_{B} T_{e}\right)^{3 / 2} F_{1 / 2}\left(\frac{\mu_{e}}{k_{B} T_{e}}\right)
$$

the heat capacity can be written as:

$$
C_{v}=\frac{3}{2} k_{B} n_{e}\left[\frac{5 F_{3 / 2}\left(\mu_{e} / k_{B} T_{e}\right)}{3 F_{1 / 2}\left(\mu_{e} / k_{B} T_{e}\right)}+T_{e} \frac{\partial}{\partial T_{e}}\left(\frac{\mu_{e}}{k_{B} T_{e}}\right)\right]
$$

Because $n_{e}$ and $T_{e}$ are independent variables, the derivative of $n_{e}$ with respect to $T_{e}$ is equal to 0 . Thus, by calculating explicitly this derivative from Eq. B5], we obtain:

$$
\frac{\partial}{\partial T_{e}}\left(\frac{\mu_{e}}{k_{B} T_{e}}\right)=-\frac{3}{T_{e}} \frac{F_{1 / 2}\left(\mu_{e} / k_{B} T_{e}\right)}{F_{-1 / 2}\left(\mu_{e} / k_{B} T_{e}\right)}
$$

and the heat capacity of electrons is finally given by:

$$
C_{v}=\frac{3}{2} k_{B} n_{e}\left[\frac{5 F_{3 / 2}\left(\mu_{e} / k_{B} T_{e}\right)}{3 F_{1 / 2}\left(\mu_{e} / k_{B} T_{e}\right)}-3 \frac{F_{1 / 2}\left(\mu_{e} / k_{B} T_{e}\right)}{F_{-1 / 2}\left(\mu_{e} / k_{B} T_{e}\right)}\right]
$$

In the limit of the ideal gas, $\mu_{e} / k_{B} T_{e} \ll-1$. Thus the Fermi-Dirac integral can be simplified to $F_{j}(x) \approx \Gamma(j+1) e^{x}$ with $\Gamma$ the Gamma function, which leads to $C_{v}=3 k_{B} n_{e} / 2$. In the limit $\mu_{e} / k_{B} T_{e} \gg 1$, the Fermi-Dirac integrals can be simplified owing to the Sommerfeld expansion leading to the heat capacity of the free electron gas $C_{v} \approx \pi^{2} k_{B}^{2} T_{e} n_{e} / 2 \varepsilon_{F}$ [16].

Regarding the effective chemical potential $\tilde{\mu}_{e}$, it comes directly:

$$
\tilde{\mu}_{e}=\frac{3}{2} \frac{\sqrt{2} m_{e}^{3 / 2}}{\pi^{2} \hbar^{3}}\left(k_{B} T_{e}\right)^{3 / 2} F_{1 / 2}\left(\frac{\mu_{e}}{k_{B} T_{e}}\right) \frac{\partial \mu_{e}}{\partial n_{e}}
$$

inserting Eq. (B2) into Eq. (A6) and using the property $\mathrm{d} F_{j}(x) / \mathrm{d} x=j F_{j-1}(x)$. By calculating the derivative of $n_{e}$ with respect to itself, one obtains:

$$
\frac{\partial n_{e}}{\partial n_{e}}=\frac{\sqrt{2} m_{e}^{3 / 2}}{\pi^{2} \hbar^{3}} \frac{k_{B} T_{e}}{2} F_{-1 / 2}\left(\frac{\mu_{e}}{k_{B} T_{e}}\right) \frac{\partial \mu_{e}}{\partial n_{e}}
$$

Obviously, this derivative is also equal to unity and it is possible to have an expression of $\partial \mu_{e} / \partial n_{e}$. Inserting this expression into Eq. [B9], the effective chemical potential is finally given by:

$$
\tilde{\mu}_{e}=3 k_{B} T_{e} \frac{F_{1 / 2}\left(\mu_{e} / k_{B} T_{e}\right)}{F_{-1 / 2}\left(\mu_{e} / k_{B} T_{e}\right)}
$$

\section{Appendix C: Electron-phonon energy transfer}

Following the theory developed by Allen [40], the energy transfer resulting from a collision between an electron and an acoustic or optical phonon is defined by:

$$
\frac{\partial E_{e}}{\partial t}=\frac{4 \pi}{\hbar} \sum_{\mathbf{k}, \mathbf{k}} \hbar \omega_{\mathbf{q}}\left|M_{\mathbf{k}, \mathbf{k}^{\prime}}\right|^{2} S\left(\mathbf{k}, \mathbf{k}^{\prime}\right) \delta\left(\varepsilon_{\mathbf{k}}-\varepsilon_{\mathbf{k}}, \hbar \omega_{\mathbf{q}}\right)
$$

The term $S\left(\mathbf{k}, \mathbf{k}^{\prime}\right)$ corresponds to the so-called thermal factor, $\varepsilon_{\mathbf{k}}$ and $\varepsilon_{\mathbf{k}}$, are electron energies in states $\mathbf{k}$ and $\mathbf{k}$ ', respectively, and $\hbar \omega_{\mathbf{q}}$ is the phonon energy absorbed or emitted by the electron moving from the state $\mathbf{k}$ to the state $\mathbf{k}$ ', and satisfying the momentum conservation $\mathbf{k}^{\prime}=\mathbf{k}+\mathbf{q}$. As the matrix element $\left|M_{\mathbf{k}, \mathbf{k}},\right|^{2}$ given by [41]:

$$
\left|M_{\mathbf{k}, \mathbf{k}}\right|^{2}=-\frac{\hbar}{2 \rho V \omega_{\mathbf{q}}}\left(n_{\mathbf{q}}+1\right) q^{2} \mathscr{U}^{2}\left(\omega_{\mathbf{q}}, \mathbf{q}\right) \delta\left(\mathbf{k}-\mathbf{k}^{\prime}+\mathbf{q}\right)
$$

with $n_{\mathbf{q}}=\left[\exp \left(\hbar \omega_{\mathbf{q}} / k_{B} T_{i l}\right)-1\right]^{-1}$ the Bose-Einstein distribution and $\mathscr{U}\left(\omega_{\mathbf{q}}, \mathbf{q}\right)$ the deformation potential. By writing the energy conservation as:

$$
\delta\left(\varepsilon_{\mathbf{k}}-\varepsilon_{\mathbf{k}},+\hbar \omega_{\mathbf{q}}\right)=\frac{m_{e}}{\hbar^{2} k q} \delta\left(\cos \theta-\frac{m_{e} \omega_{\mathbf{q}}}{\hbar k q}-\frac{q}{2 k}\right)
$$

with $\theta$ the angle between $\mathbf{k}$ and $\mathbf{q}$, where it was used the momentum conservation $\mathbf{k}^{\prime}=\mathbf{k}+\mathbf{q}$ and assumed a free electron gas, i.e. $\varepsilon_{k}=\hbar^{2} \mathbf{k}^{2} / m_{e}$. This allows to write Eq. [C1] as follows:

$$
\frac{\partial \mathscr{E}_{e}}{\partial t}=-\frac{2 \pi}{\left(8 \pi^{3}\right)^{2}} \int\left\{\int\left[\frac{m_{e} q}{\rho \hbar k}\left(n_{\mathbf{q}}+1\right) \mathscr{U}^{2}\left(\omega_{\mathbf{q}}, \mathbf{q}\right) S\left(\mathbf{k}, \mathbf{k}^{\prime}\right) \delta\left(\cos \theta-\frac{m_{e} \omega_{\mathbf{q}}}{\hbar k q}-\frac{q}{2 k}\right)\right] \mathrm{d} \mathbf{k},\right\} \mathrm{d} \mathbf{k}
$$

Note that $\sum_{\mathbf{k}}=\left(V / 8 \pi^{3}\right) \int \mathrm{d} \mathbf{k}$ has been used assuming close enough electron states and the electron energy $E_{e}$ has been replaced by the electron energy per unit volume $\mathscr{E}_{e}=E_{e} / V$. The thermal factor $S\left(\mathbf{k}, \mathbf{k}^{\prime}\right)$ is defined by $S\left(\mathbf{k}, \mathbf{k}^{\prime}\right)=\left[f\left(\varepsilon_{\mathbf{k}}\right)-f\left(\varepsilon_{\mathbf{k}^{\prime}}\right)\right] n_{\mathbf{q}}-$ 
$f\left(\varepsilon_{\mathbf{k}},\right)\left[1-f\left(\varepsilon_{\mathbf{k}}\right)\right]$ with $f\left(\varepsilon_{\mathbf{k}}\right)=\left[\exp \left(\left(\varepsilon_{\mathbf{k}}-\mu_{e}\right) / k_{B} T_{e}\right)+1\right]^{-1}$ the Fermi-Dirac distribution but can be written as:

$$
S\left(\mathbf{k}, \mathbf{k}^{\prime}\right)=\left[f\left(\varepsilon_{k}\right)-f\left(\varepsilon_{k}+\hbar \omega_{\mathbf{q}}\right)\right]\left[n_{\mathbf{q}}\left(T_{i l}\right)-n_{\mathbf{q}}\left(T_{e}\right)\right]
$$

so that it clearly appears $S\left(\mathbf{k}, \mathbf{k}^{\prime}\right)=0$ and $\partial_{t} E_{e}=0$ when $T_{e}=T_{i l}$.

Owing to momentum conservation, integration over $\mathbf{k}$ ' can be replaced by integration over $\mathbf{q}$ which gives:

$$
\int \mathrm{d}^{\prime}=\int \mathrm{d} \mathbf{q}=2 \pi \int_{0}^{+\infty} \int_{-1}^{1} q^{2} \mathrm{~d} q \mathrm{~d} \cos \theta
$$

and Eq. (C4) can be simplified after integration over $\cos \theta$ in terms of the Heaviside step function $\Theta$ as follows:

$$
\begin{aligned}
\frac{\partial \mathscr{E}_{e}}{\partial t}= & \frac{1}{16 \pi^{4}} \int\left\{\int _ { 0 } ^ { + \infty } \left[\frac{m_{e} q^{3}}{\rho \hbar k}\left(n_{\mathbf{q}}+1\right) \mathscr{U}^{2}\left(\omega_{\mathbf{q}}, \mathbf{q}\right)\left[f\left(\varepsilon_{k}\right)-f\left(\varepsilon_{k}+\hbar \omega_{\mathbf{q}}\right)\right]\left[n_{\mathbf{q}}\left(T_{e}\right)-n_{\mathbf{q}}\left(T_{i l}\right)\right]\right.\right. \\
& \left.\left.\times \Theta\left(1-\frac{m_{e} \omega_{\mathbf{q}}}{\hbar k q}-\frac{q}{2 k}\right) \Theta\left(1+\frac{m_{e} \omega_{\mathbf{q}}}{\hbar k q}+\frac{q}{2 k}\right)\right] \mathrm{d} q\right\} \mathrm{d} \mathbf{k}
\end{aligned}
$$

Acoustic phonon:

For acoustic phonons, the relation dispersion leads to $\omega_{\mathbf{q}}=c_{s} q$ with $c_{s}$ the speed of sound and the deformation potential is a constant [41] $\mathscr{U}\left(\omega_{\mathbf{q}}, \mathbf{q}\right)=\mathscr{U}$. Thus, Eq. (C7) becomes:

$$
\frac{\partial \mathscr{E}_{e}}{\partial t}=\frac{1}{16 \pi^{4}} \frac{m_{e} \mathscr{U}^{2}}{\rho \hbar} \int\left\{\frac{1}{k} \int_{0}^{2 k+2 m_{e} c_{s} / \hbar} q^{3}\left[n\left(\hbar c_{s} q, T_{i l}\right)+1\right]\left[f\left(\varepsilon_{k}\right)-f\left(\varepsilon_{k}+\hbar c_{s} q\right)\right]\left[n\left(\hbar c_{s} q, T_{e}\right)-n\left(\hbar c_{s} q, T_{i l}\right)\right] \mathrm{d} q\right\} \mathrm{d} \mathbf{k}
$$

As it appears that the integral over $\mathbf{k}$ depends only on its modulus, one has $\int \mathrm{d} \mathbf{k}=4 \pi \int_{0}^{+\infty} k^{2} \mathrm{~d} k$ and the previous equation can be simplified into:

$$
\frac{\partial \mathscr{E}_{e}}{\partial t}=\frac{1}{4 \pi^{3}} \frac{m_{e} \mathscr{U}^{2}}{\rho \hbar} \int_{0}^{k_{B Z}}\left\{k \int_{0}^{2 k+2 m_{e} c_{s} / \hbar} q^{3}\left[n\left(\hbar c_{s} q, T_{i l}\right)+1\right]\left[f\left(\varepsilon_{k}\right)-f\left(\varepsilon_{k}+\hbar c_{s} q\right)\right]\left[n\left(\hbar c_{s} q, T_{e}\right)-n\left(\hbar c_{s} q, T_{i l}\right)\right] \mathrm{d} q\right\} \mathrm{d} \mathbf{k}
$$

where the integration is restricted to the first Brillouin zone as during the solid-to-plasma transition, the electron energy does not exceed $E\left(k_{B Z}\right)$. In the limit of small momentum transfer, one has $\hbar c_{s} q \ll k_{B} T_{i l}$ and the Bose-Einstein distribution simplifies as $n\left(T_{i l}\right)+1 \approx k_{B} T_{i l} / \hbar c_{s} q$ and $n\left(T_{e}\right)-n\left(T_{i l}\right) \approx k_{B}\left(T_{e}-T_{i l}\right) / \hbar c_{s} q$. Thus, Eq. C9) becomes:

$$
\frac{\partial \mathscr{E}_{e}}{\partial t}=\frac{1}{4 \pi^{3}} \frac{m_{e} k_{B}^{2} \mathscr{U}^{2} T_{i l}}{\rho c_{s}^{2} \hbar^{3}}\left(T_{e}-T_{i l}\right) \int_{0}^{k_{B Z}}\left\{k \int_{0}^{2 k+2 m_{e} c_{s} / \hbar} q\left[f\left(\varepsilon_{k}\right)-f\left(\varepsilon_{k}+\hbar c_{s} q\right)\right] \mathrm{d} q\right\} \mathrm{d} k
$$

Finally, the energy transfer can be written in such a way that the electron-acoustic phonon energy transfer rate $v_{e t}$ appears:

$$
\frac{\partial \mathscr{E}_{e}}{\partial t}=v_{e t} n_{e} k_{B}\left(T_{e}-T_{i l}\right)
$$

where $v_{e t}$ is defined by:

$$
v_{e t}=\frac{1}{n_{e}} \frac{m_{e} k_{B} \mathscr{U}^{2} T_{i l}}{4 \pi^{3} \rho c_{s}^{2} \hbar^{3}} \int_{0}^{k_{B Z}} k \times I\left(2 k+2 \frac{m_{e} c_{s}}{\hbar}\right) \mathrm{d} k
$$

The function $I$ corresponds to the integral over $q$ in Eq. C10, and can be calculated analytically, leading to:

$$
I(\kappa)=\frac{\kappa^{2}}{2}-\frac{k_{B} T_{i l}}{\hbar c_{s}} \kappa \log \left[1+\exp \left(\frac{\varepsilon_{\kappa}+\hbar c_{s} \kappa-\mu_{e}}{k_{B} T_{i l}}\right)\right]+\frac{k_{B}^{2} T_{i l}^{2}}{\hbar^{2} c_{s}^{2}}\left[F_{1}\left(\frac{\varepsilon_{\kappa}+\hbar c_{s} \kappa-\mu_{e}}{k_{B} T_{i l}}\right)-F_{1}\left(\frac{\varepsilon_{\kappa}-\mu_{e}}{k_{B} T_{i l}}\right)\right]
$$

with $\varepsilon_{\kappa}=\hbar^{2} \kappa^{2} / 2 m_{e} . F_{1}$ is the Fermi-Dirac integral of order 1 .

Optical phonon

For optical phonons, the dispersion relation leads to $\omega_{\mathbf{q}}=\omega_{L O}$ and the deformation potential is given by the Fröhlich formalism [41-43]:

$$
\mathscr{U}^{2}\left(\omega_{\mathbf{q}}, \mathbf{q}\right)=4 \pi e^{2} \rho \omega_{L O}^{2}\left(\frac{1}{\varepsilon_{r}^{\infty}}-\frac{1}{\varepsilon_{r}^{0}}\right) \frac{1}{q^{4}}
$$


where $\varepsilon_{r}^{0}$ and $\varepsilon_{r}^{\infty}$ are the dielectric constants at low and high frequencies, respectively. Plugging them into Eq. (C7) gives after integration over $q$ :

$$
\frac{\partial \mathscr{E}_{e}}{\partial t}=\frac{1}{4 \pi^{3}} \frac{m_{e} e^{2} \omega_{L O}^{2}}{\hbar}\left(\frac{1}{\varepsilon_{r}^{\infty}}-\frac{1}{\varepsilon_{r}^{0}}\right)\left[n\left(T_{i l}\right)+1\right]\left[n\left(T_{e}\right)-n\left(T_{i l}\right)\right] \int\left\{\frac{1}{k}\left[f\left(\varepsilon_{k}\right)-f\left(\varepsilon_{k}+\hbar \omega_{L O}\right)\right] \ln \left(\frac{k+\sqrt{k^{2}-2 m_{e} \omega_{L O} / \hbar}}{k-\sqrt{k^{2}-2 m_{e} \omega_{L O} / \hbar}}\right)\right\} \mathrm{d} \mathbf{k}
$$

As for acoustic phonons, the integration over $\mathbf{k}$ depends only on its modulus leading, for the electron-optical phonon energy transfer, to:

$$
\frac{\partial \mathscr{E}_{e}}{\partial t}=\frac{m_{e}^{2} e^{2} \omega_{L O}^{2}}{\pi^{2} \hbar^{3}}\left(\frac{1}{\varepsilon_{r}^{\infty}}-\frac{1}{\varepsilon_{r}^{0}}\right)\left[n\left(T_{i l}\right)+1\right]\left[n\left(T_{e}\right)-n\left(T_{i l}\right)\right] \int_{\hbar \omega_{L O}}^{E\left(k_{B Z}\right)}\left[f\left(\varepsilon_{k}\right)-f\left(\varepsilon_{k}+\hbar \omega_{L O}\right)\right] \ln \left(\frac{1+\sqrt{1-\hbar \omega_{L O} / \varepsilon_{k}}}{1-\sqrt{1-\hbar \omega_{L O} / \varepsilon_{k}}}\right) \mathrm{d} \varepsilon_{k}
$$

after having transformed integration over $k$ to integration over $\varepsilon_{k}$. Finally, as for acoustic phonons, it is possible to exhibit an electron-optical phonon energy transfer rate defined by:

$$
v_{e t}=\frac{1}{n_{e}} \frac{m_{e}^{2} e^{2} \omega_{L O}}{\pi^{2} \hbar^{4}}\left(\frac{1}{\varepsilon_{r}^{\infty}}-\frac{1}{\varepsilon_{r}^{0}}\right)\left(n\left(T_{i l}\right)+1\right) \int_{\hbar \omega_{L O}}^{E\left(k_{B Z}\right)}\left[f\left(\varepsilon_{k}\right)-f\left(\varepsilon_{k}+\hbar \omega_{L O}\right)\right] \ln \left(\frac{1+\sqrt{1-\hbar \omega_{L O} / \varepsilon_{k}}}{1-\sqrt{1-\hbar \omega_{L O} / \varepsilon_{k}}}\right) \mathrm{d} \varepsilon_{k}
$$

giving the following electron-optical phonon energy transfer:

$$
\frac{\partial \mathscr{E}_{e}}{\partial t}=v_{e t} \hbar \omega_{L 0} n_{e}\left[n\left(T_{e}\right)-n\left(T_{i l}\right)\right]
$$

[1] J. Nuckolls, L. Wood, A. Thiessen, and G. Zimmerman, Nature , 139 (1972).

[2] D. D. Meyerhofer, R. L. McCrory, R. Betti, T. R. Boehly, D. T. Casey, T. J. B. Collins, R. S. Craxton, J. A. Delettrez, D. H. Edgell, R. Epstein, K. A. Fletcher, J. A. Frenje, Y. Y. Glebov, V. N. Goncharov, D. R. Harding, S. X. Hu, I. V. Igumenshchev, J. P. Knauer, C. K. Li, J. A. Marozas, F. J. Marshall, P. W. McKenty, P. M. Nilson, S. P. Padalino, R. D. Petrasso, P. B. Radha, S. P. Regan, T. C. Sangster, F. H. Séguin, W. Seka, R. W. Short, D. Shvarts, S. Skupsky, J. M. Soures, C. Stoeckl, W. Theobald, and B. Yaakobi, Nuclear Fusion 51, 053010 (2011).

[3] V. N. Goncharov, T. C. Sangster, R. Betti, T. R. Boehly, M. J. Bonino, T. J. B. Collins, R. S. Craxton, J. A. Delettrez, D. H. Edgell, R. Epstein, R. K. Follett, C. J. Forrest, D. H. Froula, V. Yu. Glebov, D. R. Harding, R. J. Henchen, S. X. Hu, I. V. Igumenshchev, R. Janezic, J. H. Kelly, T. J. Kessler, T. Z. Kosc, S. J. Loucks, J. A. Marozas, F. J. Marshall, A. V. Maximov, R. L. McCrory, P. W. McKenty, D. D. Meyerhofer, D. T. Michel, J. F. Myatt, R. Nora, P. B. Radha, S. P. Regan, W. Seka, W. T. Shmayda, R. W. Short, A. Shvydky, S. Skupsky, C. Stoeckl, B. Yaakobi, J. A. Frenje, M. Gatu-Johnson, R. D. Petrasso, and D. T. Casey, Phys. Plasmas 21, 056315 (2014).

[4] R. Craxton, K. Anderson, T. Boehly, V. Goncharov, D. Harding, J. Knauer, M. McCrory, P. McKenty, D. Meyerhofer, J. Myatt, A. Schmitt, J. Sethian, R. Short, S. Skupsky, W. Theobald, W. Kruer, K. Tanaka, R. Betti, T. Collins, J. Delettrez, S. Hu, J. Marozas, A. Maximov, D. Michel, P. Radha, S. Regan, T. Sangster, W. Seka, A. Solodov, J. S. ans C. Stoeckl, and J. Zuegel, Phys. Plasmas 22, 110501 (2015).

[5] G. Duchateau, S. X. Hu, A. Pineau, A. Kar, B. Chimier, A. Casner, V. Tikhonchuk, V. N. Goncharov, P. B. Radha, and E. M. Campbell, Phys. Rev. E 100, 033201 (2019).
[6] R. Ishizaki and K. Nishihara, Phys. Rev. Lett. 78, 1920 (1997).

[7] S. Bodner, Phys. Rev. Lett. 33, 761 (1974).

[8] P. B. Radha, V. N. Goncharov, T. J. B. Collins, J. A. Delettrez, Y. Elbaz, V. Y. Glebov, R. L. Keck, D. E. Keller, J. P. Knauer, J. A. Marozas, F. J. Marshall, P. W. McKenty, D. D. Meyerhofer, S. P. Regan, T. C. Sangster, D. Shvarts, S. Skupsky, Y. Srebro, R. P. J. Town, and C. Stoeckl, Phys. Plasmas 12, 032702 (2005).

[9] S. X. Hu, P. B. Radha, J. A. Marozas, R. Betti, T. J. B. Collins, R. S. Craxton, J. A. Delettrez, D. H. Edgell, R. Epstein, V. N. Goncharov, I. V. Igumenshchev, F. J. Marshall, R. L. McCrory, D. D. Meyerhofer, S. P. Regan, T. C. Sangster, S. Skupsky, V. A. Smalyuk, Y. Elbaz, and D. Shvarts, Phys. Plasmas 16, 112706 (2009).

[10] S. X. Hu, L. A. Collins, V. N. Goncharov, J. D. Kress, R. L. McCrory, and S. Skupsky, Phys. Plasmas 23, 042704 (2016).

[11] J. L. Peebles, S. X. Hu, W. Theobald, V. N. Goncharov, N. Whiting, P. M. Celliers, S. J. Ali, G. Duchateau, E. M. Campbell, T. R. Boehly, and S. P. Regan, Phys. Rev. E 99, 063208 (2019).

[12] A. Pineau, B. Chimier, S. X. Hu, and G. Duchateau, Phys. Plasmas 27, 092703 (2020).

[13] Y. Lee and R. More, Phys. Fluids 27 (1984).

[14] C. A. Ordonez and M. I. Molina, Phys. Rev. Lett. 72, 2407 (1994).

[15] U. Gaur and B. Wunderlich, J. Phys. Chem. Ref. Data 11, 313 (1982).

[16] N. Ashcroft and N. Mermin, Physique des Solides (EDP Sciences, 2002).

[17] S. Atzeni and J. Meyer-ter Vehn, The Physics of Inertial Fusion (Clarendon Press, Oxford, 2004).

[18] S. X. Hu, L. A. Collins, V. N. Goncharov, J. D. Kress, R. L. 
McCrory, and S. Skupsky, Phys. Rev. E 92, 043104 (2015).

[19] V. Sangawar and M. Golchha, Int. Jour. Sci. Eng. Res. 4, 2700 (2013).

[20] B. Arad and S. Eliezer, Phys. Rev. A 18, 2261 (1978).

[21] B. Rethfeld, Phys. Rev. Lett. 92, 187401 (2004).

[22] L. V. Keldysh, Sov. Phys. JETP 20, 1307 (1965).

[23] M. Chergui, Femtochemistry, Ultrafast Chemical and Physical Processes on Molecular Systems (World Scientific, 1995).

[24] M. Gryziński, Phys. Rev. 138, A336 (1965).

[25] Y. B. Zel'dovich and Y. P. Raizer, Physics of Shock Waves and High-Temperature Hydrodynamic Phenomena, edited by W. D. Hayes and R. F. Probstein (Academic Press, 1966).

[26] E. M. Lifshitz and L. P. Pitaevskii, Course of Theoretical Physics. Volume 10: Physical Kinetics (ButterworthHeinemann, 1981).

[27] V. N. Goncharov, T. C. Sangster, T. R. Boehly, S. X. Hu, I. V. Igumenshchev, F. J. Marshall, R. L. McCrory, D. D. Meyerhofer, P. B. Radha, W. Seka, S. Skupsky, C. Stoeckl, D. T. Casey, J. A. Frenje, and R. D. Petrasso, Phys. Rev. Lett. 104, $165001(2010)$

[28] A. Kar, S. X. Hu, G. Duchateau, J. Carroll-Nellenback, and P. B. Radha, Phys. Rev. E 101, 063202 (2020).

[29] D. H. Edgell, W. Seka, R. E. Bahr, T. R. Boehly, and M. J. Bonino, Phys. Plasmas 15, 092704 (2008).

[30] L. Spitzer, Physics of Fully Ionized Gases (Interscience Publishers Inc., 1956).

[31] W. Kruer, The Physics Of Laser Plasma Interactions (CRC Press, 2003).

[32] J. D. Lindl, Phys. Plasmas 2, 3933 (1995).

[33] V. N. Goncharov, J. P. Knauer, P. W. McKenty, P. B. Radha, T. C. Sangster, S. Skupsky, R. Betti, R. L. McCrory, and D. D.
Meyerhofer, Phys. Plasmas 10, 1906 (2003).

[34] S. X. Hu, V. N. Goncharov, T. R. Boehly, R. L. McCrory, S. Skupsky, L. A. Collins, J. D. Kress, and B. Militzer, Phys. Plasmas 22, 056304 (2015).

[35] J. Oh, A. J. Schmitt, M. Karasik, and S. P. Obenschain, Phys. Plasmas 28, 032704 (2021).

[36] H. Nishimura, H. Shiraga, H. Azechi, N. Miyanaga, M. Nakai, N. Izumi, M. Nishikino, M. Heya, K. Fujita, Y. Ochi, K. Shigemori, N. Ohnishi, M. Murakami, K. Nishihara, R. Ishizaki, H. Takabe, K. Nagai, T. Norimatsu, M. Nakatsuka, T. Yamanaka, S. Nakai, C. Yamanaka, and K. Mima, Nuclear Fusion 40, 547 (2000).

[37] S. Depierreux, C. Labaune, D. T. Michel, C. Stenz, P. Nicolaï, M. Grech, G. Riazuelo, S. Weber, C. Riconda, V. T. Tikhonchuk, P. Loiseau, N. G. Borisenko, W. Nazarov, S. Hüller, D. Pesme, M. Casanova, J. Limpouch, C. Meyer, P. Di-Nicola, R. Wrobel, E. Alozy, P. Romary, G. Thiell, G. Soullié, C. Reverdin, and B. Villette, Phys. Rev. Lett. 102, 195005 (2009).

[38] S. Y. Guskov, J. Limpouch, P. Nicolaï, and V. T. Tikhonchuk, Physics of Plasmas 18, 103114 (2011).

[39] L. D. Landau and E. M. Lifshitz, Course of Theoretical Physics. Volume 5: Statistical Physics 3rd Edition Part 1 (ButterworthHeinemann, 1980).

[40] P. Allen, Phys. Rev. Lett. 59, 1460 (1987).

[41] J. Ziman, Electrons and Phonons, edited by N. Mott, E. Bullard, and D. Wilkinson (Clarendon Press, 1960).

[42] H. Fröhlich, H. Pelzer, and S. Zienau, Philosophical Magazine Series 7 41, 221 (1950).

[43] F. Guistino, Rev. Mod. Phys. (2017). 